\title{
Molecular Diagnosis of Sexually Transmitted Chlamydia trachomatis in the United States
}

\author{
April L. Harkins ${ }^{1}$ and Erik Munson ${ }^{2,3}$ \\ ${ }^{1}$ Department of Clinical Laboratory Science, Marquette University, Milwaukee, WI 53233, USA \\ ${ }^{2}$ Wheaton Franciscan Laboratory, 11020 West Plank Court, Suite 100, Wauwatosa, WI 53226, USA \\ ${ }^{3}$ College of Health Sciences, University of Wisconsin-Milwaukee, Milwaukee, WI 53201, USA
}

Correspondence should be addressed to Erik Munson, erik.munson@wfhc.org

Received 4 March 2011; Accepted 27 April 2011

Academic Editor: E. Petru

Copyright ( 2011 A. L. Harkins and E. Munson. This is an open access article distributed under the Creative Commons Attribution License, which permits unrestricted use, distribution, and reproduction in any medium, provided the original work is properly cited.

Chlamydia, with its Chlamydia trachomatis etiology, is the most common bacterial sexually transmitted infection in the United States and is often transmitted via asymptomatic individuals. This review summarizes traditional and molecular-based diagnostic modalities specific to $C$. trachomatis. Several commercially available, FDA-approved molecular methods to diagnose urogenital $C$. trachomatis infection include nucleic acid hybridization, signal amplification, polymerase chain reaction, strand displacement amplification, and transcription-mediated amplification. Molecular-based methods are rapid and reliable genital specimen screening measures, especially when applied to areas of high disease prevalence. However, clinical and analytical sensitivity for some commercial systems decreases dramatically when testing urine samples. In vitro experiments and clinical data suggest that transcription-mediated amplification has greater analytical sensitivity than the other molecular-based methods currently available. This difference may be further exhibited in testing of extragenital specimens from at-risk patient demographics. The development of future molecular testing could address conundrums associated with confirmatory testing, medicolegal testing, and test of cure.

\section{Ecology and Epidemiology of Urogenital Chlamydia}

1.1. Prevalence and Transmission. Since becoming a nationally notifiable disease in the United States in 1995, chlamydia has experienced consistent annual increases (averaging $5.8 \%$ ) to its 2009 prevalence rate of 409.2 per 100,000 inhabitants (Figure 1(a)), making it the most common bacterial sexually transmitted infection (STI) in this country [1]. Data from the United States Centers for Disease Control and Prevention (CDC) have revealed that chlamydia rates are highest in late adolescents and young adults ([1], Figure 1(b)). African Americans and Native Americans demonstrate higher rates of chlamydia compared to other races or ethnicities (Figure $1(\mathrm{c}))$.

Sexually active asymptomatic populations have been implicated in widespread transmission of the Chlamydia trachomatis etiology. Selective screening of sexually active women has yielded infection rates ranging from $8 \%$ to $40 \%$ (typical mean of $15 \%,[2]$ ), while approximately $10 \%$ of sexually active asymptomatic males are infected $[3,4]$. In contrast to Neisseria gonorrhoeae infection in which most patients develop symptoms and seek care promptly, Hook et al. [5] reported that most females and males with $C$. trachomatis infection were asymptomatic or mildly symptomatic upon clinical presentation. Diagnosis was largely on the basis of screening or having a symptomatic contact. Further evidence that chlamydia is a prevalent disease rather than an incident disease comes from extrapolations of STI agent acquisition rates. While past studies have suggested that gonorrhea sexual transmission can be more efficient than chlamydia transmission $[6,7]$, recent data utilizing $C$. trachomatis molecular diagnostics report less of a disparity between transmission rates [8]. Katz et al. [7] estimated a $0.32-0.39$ chlamydia transmission rate when using culture as a detection modality, while Quinn et al. [8] estimated this rate to be approximately 0.68 when utilizing molecular diagnostics. It is important to note that these extrapolations 


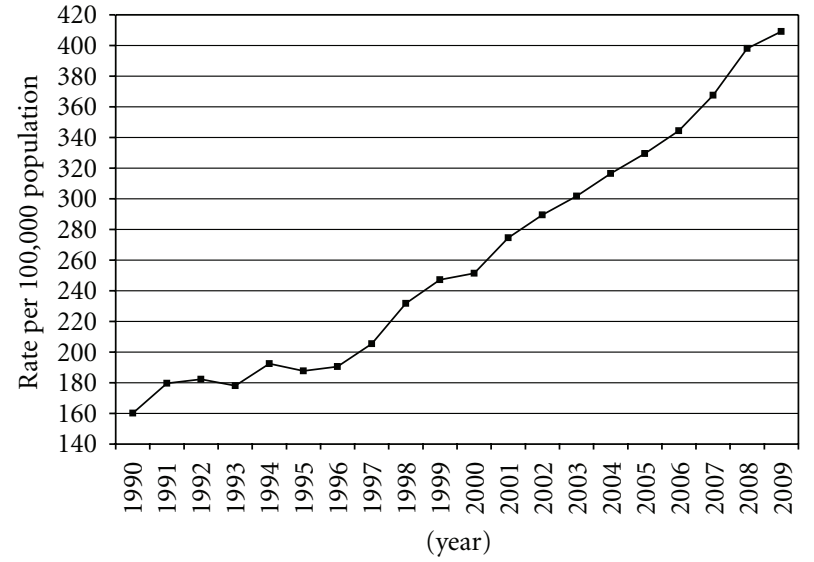

(a)

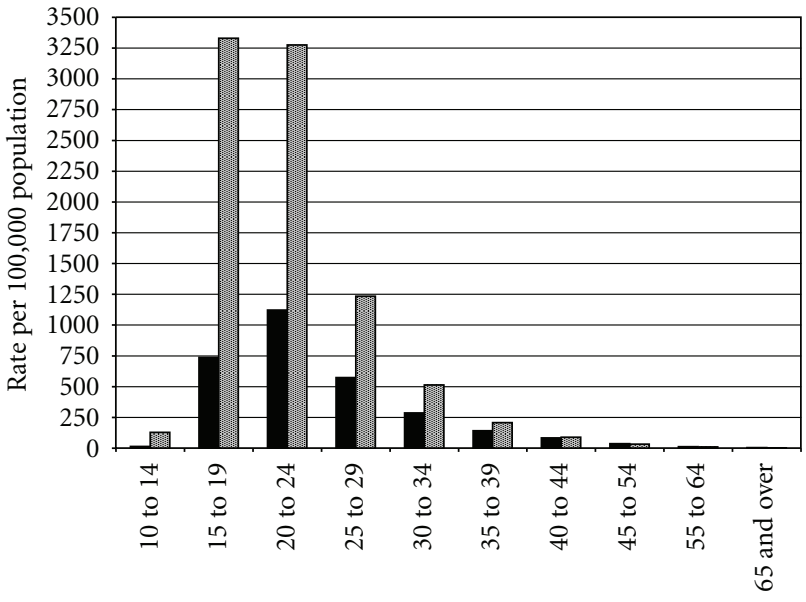

Age group

(b)

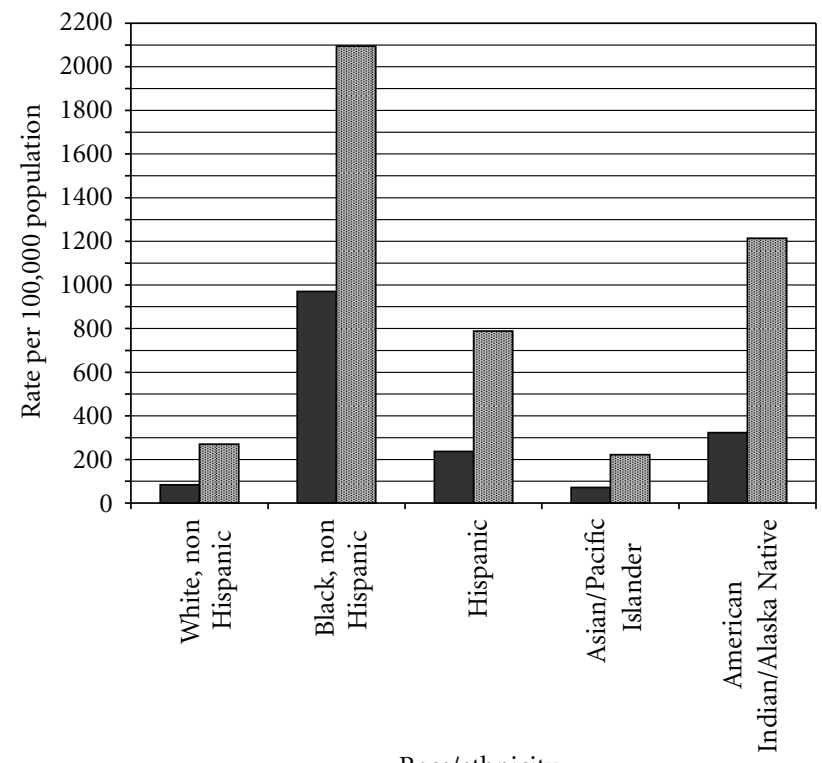

Race/ethnicity

(c)

FIgURE 1: Epidemiology of chlamydia in the United States, summarized in terms of annual incidence rates (a), prevalence within arbitrary age groups (b), and race/ethnicity distribution (c). Solid bars represent male gender and shaded bars represent female gender. Data are adapted from $[1]$.

were performed on the basis of historical average frequency of intercourse between pairs, rather than single sexual encounter, which provided the basis for studies of gonorrhea transmission $[9,10]$.

1.2. Urogenital Chlamydia in Males. Ocular trachoma, lymphogranuloma venereum, perinatal infections, and adult oculogenital disease outline four clinical categories of $C$. trachomatis infections described by Stamm et al. [11]. We briefly summarize selected important clinical manifestations of urogenital disease as they pertain to subsequent laboratory diagnosis of the disease etiology. C. trachomatis is thought to be responsible for $30-50 \%$ of cases of nongonococcal urethritis (NGU) in men. Appropriate laboratory diagnostics in male urethritis are important for at least four reasons: (1) symptom overlap with clinical gonococcal urethritis and NGU may exist (Table 1); (2) prevalence of NGU in the United States exceeds that of gonococcal urethritis [2]; (3) C. trachomatis may be detected from a substantial proportion of patients with gonococcal urethritis [12], and concomitantly, (4) dually infected males who are treated solely for gonococcal urethritis are likely to develop post gonococcal urethritis, manifested as persistence or recurrence. Risk factors for chlamydial urethritis have included heterosexual orientation, African American race, and age younger than 20 years [13].

1.3. Urogenital Chlamydia in Females. Mucopurulent cervicitis caused by $C$. trachomatis is said to be the female 
TABLE 1: Factors that attempt to distinguish male nongonococcal urethritis from those cases caused by Neisseria gonorrhoeae.

\begin{tabular}{|c|c|c|c|}
\hline Factor & Gonococcal urethritis & Nongonococcal urethritis & Reference(s) \\
\hline Typical incubation period & $2-5$ days & $7-14$ days & {$[14,15]$} \\
\hline Range of symptom onset & $1-10$ days & $2-35$ days & {$[14,15]$} \\
\hline Frank purulent discharge ( $\%$ of cases) & 75 & $11-33$ & {$[16,17]$} \\
\hline Mucopurulent discharge ( $\%$ of cases) & 25 & 50 & {$[16,17]$} \\
\hline Clear or moderately viscid discharge ( $\%$ of cases) & 4 & $10-50$ & {$[16,17]$} \\
\hline Dysuria (\% of cases) & $73-88$ & $53-75$ & {$[16,18]$} \\
\hline Combination of dysuria and discharge (\% of cases) & 71 & 38 & {$[16]$} \\
\hline
\end{tabular}

counterpart of male NGU, as approximately $70 \%$ of women are asymptomatic or experience only mild symptoms such as bleeding, discharge, mild abdominal pain, and dysuria. Being a sex partner of a male with NGU or gonococcal urethritis has been reported to confer an infection risk of greater than $30 \%[2,12]$. Additional factors such as younger age, African American race, unmarried status, new or multiple sex partners, oral contraceptive use, and residence in the southeast United States promote higher rates of chlamydia in sexually active females $[2,19]$.

Symptoms relative to mucopurulent cervicitis can also characterize conditions such as cystitis and vaginitis. As such, diagnosis of chlamydia may be masked by diagnosis and treatment of concomitant N. gonorrhoeae and Trichomonas vaginalis infection. A Milwaukee, Wisconsin laboratory used highly sensitive molecular methods for the detection of these agents $[20,21]$ to determine the STI profile of 272 female healthcare encounters that proved to be positive for at least one STI. In this populace that ranks second in the United States in both chlamydia and gonorrhea prevalence [1], 17\% of patients with detectable C. trachomatis-specific nucleic acid had concomitant $N$. gonorrhoeae and/or $T$. vaginalis nucleic acid detection [22]. The development of accurate laboratory diagnostics for $C$. trachomatis bears additional importance in light of data associating chlamydial cervicitis with acquisition of human immunodeficiency virus in women $[23,24]$.

\section{Selected Nonmolecular Means of Laboratory Diagnosis}

For many years, the accepted gold standard for C. trachomatis detection was culture. Culturing techniques in McCoy cell lines are rather complex and time consuming, with the necessity for experienced laboratory technologists for accurate follow-up staining and microscopy. Sensitivity of culture methodology is much less compared to nucleic acid amplification testing (NAAT), allowing for false negative results to potentiate the spread of infection [25-28].

In 2002, the CDC recommended routine laboratory screening for individuals at high risk of acquiring STIs [29], particularly with effective treatment regimens for $C$. trachomatis and $N$. gonorrhoeae being both accessible and inexpensive. The detection of $C$. trachomatis by rapid screening or point-of-care methods includes nonamplification methods such as direct fluorescent antibody testing (DFA), optical immunoassay (OIA), and rapid solid-phase enzyme immunoassay (EIA). These methods were developed to provide a level of service to the community, enabling clinicians to begin treating patients on the day of the detection (a "test and treat" strategy) and consequently reducing the risk of inflammatory sequelae and the spread of infection. This represents a significant public health issue as $20 \%$ of patients that are diagnosed with $C$. trachomatis do not return to follow-up medical attention within a one-month interval, with $3 \%$ of these patients subsequently developing PID within this timeframe $[30,31]$.

The DFA procedure begins with fixing epithelial cells from the conjunctiva, urethra, or cervix to a microscope slide. Monoclonal antibodies specific for C. trachomatis major outer membrane protein, conjugated with fluorescein isothiocyanate (FITC), bind to intracellular inclusions if the organism is present. The DFA procedure is considered rapid or point-of-care testing due to its capacity to be performed within 30 minutes, although expertise in reading fluorescence microscopy is required and the method exhibits low sensitivity when compared to NAAT. In one study, Boyadzhyan et al. [32] reported that C. trachomatis culture and DFA failed to detect $28 \%$ and $0 \%$, respectively, of specimens determined to be positive by NAAT.

The rapid OIA consists of an optical reading, which has a very subjective color change as its basis. C. trachomatis antigens present within specimens will react with specific antibodies impregnated on a silicon wafer. The Biostar OIA has been evaluated in urogenital specimens from women at an STI clinic [25, 26]. Swain et al. [25] evaluated 1,385 women for $C$. trachomatis infection using the DFA, OIA, and culture methods. Sensitivity and specificity for testing methods were $73.6 \%$ and $99.9 \%$, respectively, for DFA, $64.2 \%$ and $99.1 \%$ for OIA, $56.1 \%$ and $100 \%$ for culture, and $95.3 \%$ and $99.8 \%$ for PCR. During the study, these modalities were referenced against an expanded gold standard, which included NAAT testing if the culture was negative. The researchers concluded that a universal screening program utilizing rapid testing for laboratory diagnosis of C. trachomatis was not recommended. The decision analysis the investigators provided did show that the poorly sensitive rapid testing is potentially useful in clinics where patients do not comply to follow-up treatments.

The true sensitivity of nonmolecular diagnostic testing for C. trachomatis is predicated on the quality of the reference standard. Those assays that are compared to culture 
or analogous rapid or point-of-care testing in terms of sensitivity could demonstrate falsely elevated performance characteristics. Bandea and colleagues [27] showed the sensitivity of the Biostar Chlamydia OIA as $78.6 \%$ and specificity as $97.2 \%$ with culture as the reference standard. This is in contrast to another study [33], utilizing a reference standard based on concordant results from two NAAT modalities that reported sensitivity and specificity for the Biostar OIA at 59.4\% and 98.4\%, respectively. Moreover, $C$. trachomatis detection by point-of-care EIA methodology has also shown to have decreased sensitivity compared to NAAT. Van Dommelen et al. [34] demonstrated that sensitivity of three rapid EIAs was extremely low (17.1\% to $25 \%$ ) compared to NAAT.

Using a test with a low sensitivity may result in patients being falsely reassured by a negative test result, potentiating spread of infection and progression of disease to PID or other infertility sequelae [35]. In assessing the proper rapid test to use for $C$. trachomatis diagnosis, the World Health Organization has recently released the ASSURED criteria for rapid STI assays; affordable, sensitive, specific, userfriendly, rapid and robust, equipment-free and deliverable [35]. The optimal context for utilization of point-of-care testing is reflexive followup with NAAT. Until sensitivity of rapid point-of-care testing improves, one has to be very cautious in using nonamplification methods alone, especially in low-prevalence populations in which assays yield positive predictive value of $<90 \%$.

\section{Molecular Means of Laboratory Diagnosis}

3.1. Commercial Nucleic Acid Hybridization. Nucleic acid hybridization technologies employ oligonucleotide sequences that are designed to anneal to complementary sequences within target nucleic acid. Because of analytical sensitivity issues inherent to nucleic acid hybridization, this paradigm is generally reserved for clinical conditions with a high organism burden [36]. In a study set of 201 cervical specimens, LeBar et al. [37] determined the sensitivity of PACE 2 (Gen-Probe, Incorporated, San Diego, Calif, USA) for the detection of $C$. trachomatis-specific $16 \mathrm{~S}$ ribosomal RNA to be $82.8 \%$ compared to a $C$. trachomatis cell culture reference. $78.0 \%$ assay sensitivity was reported in an additional study of 217 cervical specimens [38]. Specificity of C. trachomatisspecific PACE 2 was documented at 98.8-99.4\% [37, 38]. In a study of male urethral specimens, Kluytmans et al. [39] developed two off-label modifications to the $C$. trachomatis PACE 2 assay that yielded assay sensitivity of 89.5\%. Within 398 endocervical specimens, Limberger et al. [40] reported that 19 of 20 C. trachomatis cell culturepositive specimens were also PACE 2-positive. This high frequency of concordance was not noted with specimens that were mailed to the laboratory for analogous nucleic acid hybridization for $N$. gonorrhoeae and may therefore reflect the susceptibility of the latter STI agent to conditions of specimen transport. This may be particularly true in light of past data reporting a $99.4 \% N$. gonorrhoeae-specific PACE 2 sensitivity in a sampling of 436 cervical or urethral swabs [41]. Taken together, these data begin to portend that decreased sensitivity of nucleic acid hybridization testing for C. trachomatis may be linked with a suboptimal cell culture reference method, rather being limited by mitigating factors such as specimen transport.

3.2. Commercial Signal Amplification. Neither target nor oligonucleotide probe nucleic acid concentrations change in the signal amplification paradigm. Instead, the concentration of reporter molecules is increased at the site of target/probe hybridization [36]. Commercially available signal amplification methods detecting $C$. trachomatis alone, or in concert with $N$. gonorrhoeae, utilize hybrid capture technology (Hybrid Capture II (HC2) product line; Digene Corporation (QIAGEN), Gaithersburg, Md, USA). Targets for $C$. trachomatis the detection include specific chromosomal and cryptic plasmid sequences. Schachter et al. [42] evaluated the HC2 CT-ID test using a combined C. trachomatis cell culture and direct fluorescent antibody reference method and demonstrated $97.7 \%$ sensitivity for detection of C. trachomatis from endocervical specimens. Within the 129 truepositive specimens, only $114(88.4 \%)$ yielded a positive $C$. trachomatis culture result. The utilization of the antecedent HC2 CT/GC test to screen for the presence of either $N$. gonorrhoeae or C. trachomatis exhibited 95\% sensitivity. A two-center study [43] reported $96.6 \%$ sensitivity of the HC2 CT-ID test on endocervical specimens from high-risk female populations in relation to a culture reference. However, upon the adjudication of discrepancies with PCR, sensitivity of the HC2 CT-ID test was $97.2 \%$ compared to a culture sensitivity of $80.6 \%$. Greater than $98 \%$ specificity was noted in both studies [42, 43]. Modarress et al. [44] evaluated the HC2 CT/GC test using genital swab specimens collected for PACE 2 testing and demonstrated approximately $87 \%$ and $100 \%$ sensitivity of PACE 2 and HC2 CT/GC, respectively, for the detection of either C. trachomatis or N. gonorrhoeae, although only the difference in $C$. trachomatis detection rate was significant between the two modalities $(P<0.016)$.

3.3. Commercial Polymerase Chain Reaction (PCR). The long-standing PCR $[45,46]$ DNA target amplification method is the basis of the multiplex AMPLICOR CT/NG product line (including the COBAS fully automated platform), heretofore, referred to as AMP (Roche Molecular Systems, Incorporated, Branchburg, NJ). C. trachomatis target for this assay is a 207-nucleotide sequence within a cryptic plasmid that is highly conserved within all serotypes of the organism. Livengood III and Wrenn [28] demonstrated a disparity in the rate of $C$. trachomatis detection from endocervical specimens by AMP (93.3\%) versus C. trachomatis culture $(65.0 \%)$-far greater than that disparity $(3.7 \%)$ observed for $N$. gonorrhoeae. A multicenter evaluation of AMP yielded $89.2-89.7 \%$ sensitivity in the detection of C. trachomatis target from female urine and endocervical specimens, respectively, with $88.6-90.3 \%$ sensitivity derived from male urethral and urine specimens when using an infected patient standard [47]. Data from a European study [48] demonstrated 92.0-98.0\% AMP sensitivity from male specimen sources and female endocervical specimens, 
TABle 2: Performance characteristics of three commercially-available Chlamydia trachomatis molecular screening platforms per manufacturer-provided data. Ranges reflect differences in performance characteristics between specimens collected from symptomatic and asymptomatic individuals (when specified).

\begin{tabular}{|c|c|c|c|c|}
\hline \multirow{2}{*}{ Commercial assay } & \multirow{2}{*}{ Gender } & \multirow{2}{*}{ Specimen } & \multicolumn{2}{|c|}{ Performance indices } \\
\hline & & & Sensitivity range $(\%)$ & Specificity range (\%) \\
\hline \multirow{4}{*}{ AMPLICOR } & \multirow{2}{*}{ Female } & Endocervical & $87.4-94.0$ & 98.6 \\
\hline & & Urine & $84.3-89.5$ & $98.0-98.8$ \\
\hline & \multirow{2}{*}{ Male } & Urethral & $96.3-98.7$ & $95.2-97.7$ \\
\hline & & Urine & $87.6-92.0$ & 91.9-95.7 \\
\hline \multirow{4}{*}{ BD ProbeTec ET } & \multirow{2}{*}{ Female } & Endocervical & $88.7-96.8$ & $97.9-98.5$ \\
\hline & & Urine & $77.0-83.9$ & $98.2-98.3$ \\
\hline & \multirow{2}{*}{ Male } & Urethral & $89.5-95.5$ & $92.9-97.0$ \\
\hline & & Urine & $89.5-95.4$ & $89.4-95.8$ \\
\hline \multirow{4}{*}{ APTIMA Combo $2^{\dagger}$} & \multirow{2}{*}{ Female } & Endocervical & $92.4-98.4$ & $96.7-98.8$ \\
\hline & & Urine & $93.8-96.8$ & $98.8-99.0$ \\
\hline & \multirow{2}{*}{ Male } & Urethral & $94.6-96.4$ & $96.9-98.4$ \\
\hline & & Urine & $96.3-98.5$ & $98.4-98.8$ \\
\hline
\end{tabular}

${ }^{\dagger}$ APTIMA Combo 2 clinician-collected vaginal swab sensitivity range 96.5-96.7\%, specificity range 96.4-97.2\%; APTIMA Combo 2 patient-collected vaginal swab sensitivity $98.4 \%$, specificity $96.8 \%$.

yet reported $82.5 \%$ sensitivity of C. trachomatis detection from female urine specimens. The aforementioned studies reported $\geq 98.4 \%$ specificity from all specimen sources [ 47 , 48]. From a peripheral and foreshadowing sense, noteworthy from the van Doornum et al. data [48] and an additional study [49] was the apparent deficit of AMP to accurately detect $N$. gonorrhoeae DNA from female urine specimens (sensitivity values ranged from 64.8-66.7\%). A summary of manufacturer-published performance characteristics of commercial NAAT on urine and endocervical/urethral specimens described in this review is presented in Table 2 [50-52].

3.4. Commercial Strand Displacement Amplification (SDA). A multiplex isothermal DNA target amplification method [53] constitutes a leading diagnostic assay for the detection of C. trachomatis in the United States (BD ProbeTec ET C. trachomatis and N. gonorrhoeae amplified DNA assay (heretofore referred to as ProbeTec); Becton, Dickinson and Company, Sparks, Md, USA). In the context of C. trachomatis, SDA targets the chlamydial cryptic plasmid - up to ten copies of which can be found in each cell. A seven-center evaluation [54] utilized C. trachomatis-specific cell culture, DFA, and a since-defunct commercial ligase chain reaction to determine infected patient status and related 92.5\% ProbeTec sensitivity in the detection of C. trachomatis from male urethral swabs. Sensitivity of the assay on male urine (93.1\%) exceeded that of the commercial PCR assay described previously [47]. In spite of reasonable sensitivity for the detection of $C$. trachomatis derived from endocervical swabs $(92.8 \%)$, assay of female urine yielded only $80.5 \%$ sensitivity. However, combined percentage specificity of the assay was high among both genders $(97.3 \%$, [54]) yet it is noteworthy that the specificity of urine specimens among 124 symptomatic males with positive infection status was $92.6 \%$. The analogous value for urethral specimens was $95.9 \%$.
3.5. Commercial Transcription-Mediated Amplification (TMA). A third commonly utilized nucleic acid target amplification method in the United States for the detection of $C$. trachomatis has its basis in isothermal TMA [55]. A $10^{9}$-fold rate of RNA amplification is reported to occur in two hours via TMA [56] in contrast to a $10^{6}$-fold DNA amplification rate in three to four hours [46]. The multiplex Gen-Probe APTIMA Combo 2 assay (heretofore referred to as AC2) targets C. trachomatis-specific $23 \mathrm{~S}$ ribosomal (r)RNA which is present in high copy number. A seven-site evaluation of 1391 females [21] demonstrated $94.2 \%$ C. trachomatis assay sensitivity from endocervical specimens. Sensitivity of C. trachomatis detection from female urine $(94.7 \%)$ was markedly higher than those values derived from AMP or ProbeTec. In this study, specificity of AC2 ranged from $97.6 \%$ for endocervical specimens to $98.9 \%$ for urine specimens. In response to commercial systems, especially AMP, demonstrating nonspecific amplification in the context of $N$. gonorrhoeae NAAT [57-59], direct challenges of AC2 with nonpathogenic Neisseria spp. and chlamydiae other than $C$. trachomatis failed to result in amplification [60]. Furthermore, Lowe et al. [61] noted a 10\% greater sensitivity of AC2 than AMP for detecting C. trachomatis in urine specimens.

\section{Comparison of Performance Characteristics of Commercial NAAT}

4.1. Analytical Sensitivity. The increased clinical sensitivity exhibited by AC2 may reflect a phenomenon specific to TMA. A $34.6 \%$ serum detection rate of hepatitis $\mathrm{C}$ virus (HCV)-specific nucleic acid via TMA was demonstrated in disease relapse patients who had apparent virus clearance according to conventional qualitative and quantitative PCR assays [62]. Sarrazin et al. [63] reported a 51.1\% residual serum HCV detection rate by TMA versus conventional 
qualitative PCR assays, including a 36.4\% rate compared to an assay with a lower detection limit of 100 nucleic acid copies/mL. Chernesky et al. [64] prepared mock swab specimens containing propagated $C$. trachomatis elementary bodies and showed that the analytical sensitivity of AC2 was 1000 -fold greater than that of ProbeTec and 10-fold greater than that of AMP. AC2 exhibited 100-fold greater sensitivity than the two comparators with analogous mock urine specimens. Ikeda-Dantsuji et al. [65] dispensed standardized amounts of $C$. trachomatis elementary bodies into mock specimens and showed within a subsequent dilution series that AC2 analytical sensitivity was 1000-fold greater than that of AMP. Wood et al. [66] demonstrated a lower limit of detection of $N$. gonorrhoeae via AC2 ( $10^{2}$ colony forming units $/ \mathrm{mL}$ ) than that rendered by ProbeTec or AMP ( $\geq 10^{3}$ colony forming units $/ \mathrm{mL}$ ).

4.2. Role of Endogenous Specimen Inhibitors. A second contributory factor to the purported increased analytical sensitivity of AC2 is decreased susceptibility of the assay to endogenous inhibitors of nucleic acid amplification. Substances suggested to inhibit $C$. trachomatis NAAT have included hemoglobin, low-pH cervical mucosa, $\beta$-chorionic gonadotropin, urine crystals, and urine nitrites [67-69]. Analysis of the first-generation Gen-Probe Chlamydia TMA assay using 388 urine specimens revealed an $11.9 \%$ rate of amplification inhibition [67]. This figure exceeded that of commercial PCR by nearly 5\%. Introduction of the organism-specific nucleic acid target capture protocol with concomitant washing and aspiration (under the auspices of second-generation AC2) has negated this inhibitory effect. Ikeda-Dantsuji et al. [65] subjected mock AC2 and AMP specimens containing $C$. trachomatis near the AMP lower limit of detection to increasing concentrations of phosphate and iron and demonstrated that both chemicals only promoted an inhibitory effect on AMP performance. Gaydos et al. [70] reported 75 true-positive C. trachomatis screens from 506 total female and male urine specimens via AC2 plus an additional four specimens that also tested positive by a TMA-based assay targeting an alternative sequence. These data compared favorably to the 72 true-positive results from the same study set identified by ProbeTec.

In an ex vivo study [64], rates of C. trachomatis nucleic acid amplification inhibition for AC2 (1.3-1.7\%) were fairly equivalent to that of ProbeTec $(2.0 \%)$ for female genital swabs but were far less than those derived from AMP (10.4-12.8\%). Rates of amplification inhibition from urine specimens were exceedingly high for ProbeTec and AMP (27.2\% and $12.1 \%$, resp.), when compared to AC2 (0.3\%). Modifications to the ProbeTec urine collection and transport system have addressed issues related to amplification inhibition [71]. A recent Canadian study [72] reported 98.0\% ProbeTec sensitivity in the detection of C. trachomatis from 500 urine specimens compared to AC2 sensitivity of $99.0 \%$. Analogous sensitivity indices for the detection of N. gonorrhoeae were $95.8 \%$ for ProbeTec and 100\% for AC2. Improved performance of NAAT on urine specimens, and subsequent overall clinical acceptance of this specimen

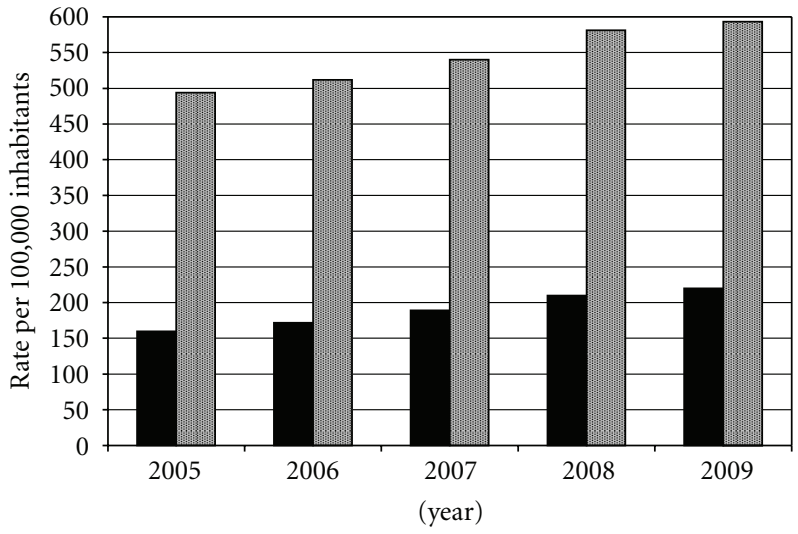

FIgURE 2: Chlamydia rates per 100,000 inhabitants by gender in the United States from 2005-2009. Filled bars represent male gender and shaded bars represent female gender. Data are adapted from [1].

source, has been cited by the CDC [1] as a factor responsible for a larger increase in $C$. trachomatis detection in males in the United States from 2005-2009 (37.6\%) than that increase observed in females (20.3\%; Figure 2).

4.3. Extragenital Specimen Sources. C. trachomatis has a tropism for columnar epithelial cells [73]. This cell type constitutes the vagina of prepubescent girls and is replaced with stratified squamous epithelium upon increased concentrations of estrogen at puberty [74]. In spite of this histological difference, $C$. trachomatis has efficiently been recovered from adult vaginal specimens, further promulgating the high analytical sensitivity of NAAT. Schachter et al. [75] utilized a variety of commercial NAAT modalities to demonstrate that vaginal swabs had nearly equivalent sensitivity to that of endocervical swabs for the detection of C. trachomatis, with approximately $12 \%$ more sensitivity than first-catch urine. Moreover, patient-collected vaginal swabs had equal sensitivity as clinician-collected vaginal swabs. A multicenter investigation of AC2 performance for C. trachomatis on vaginal swabs [76] revealed $96.6 \%$ and $96.7 \%$ sensitivity on patient- and clinician-collected specimens, respectively, extending previous findings [75]. Positive C. trachomatis vaginal screening results were in $91 \%$ and $95 \%$ concordance with those from endocervical and first-void urine collection, respectively [76]. Sensitivity of C. trachomatis AMP from a vaginal swab was $18-22 \%$ greater than that of a $C$. trachomatis EIA [77]. C. trachomatis detection via ProbeTec and AMP revealed equivalent sensitivity for both vaginal and endocervical specimens [78]. In a study of C. trachomatis detection via AC2, 98.6\% of infected women were detected via vaginal swab testing, compared to $89.9 \%$ and $81.2 \%$ from endocervical swabs and first-void urine, respectively [64]. These AC2 specimen-specific percentages of detection were statistically higher than analogous percentages generated by ProbeTec and AMP $(P=0.001)$. In a limited data set $(n=$ 25 determinations), our laboratory has demonstrated that the transfer of $200-\mu \mathrm{L}$ aliquots of vaginal saline suspensions (originally designated for microscopic examination of vulvovaginitis etiologies) into AC2 specimen transport tubes (lysis 
medium) results in the detection of $C$. trachomatis upon performance of AC2 (E. Munson, unpublished observations). To date, AC2 is the only commercially available modality that has received an FDA indication for vaginal swab collections.

In a description of a potential role for NAAT in laboratory diagnosis of rectal chlamydia, Schachter et al. [79] reported an $\geq 36.7 \%$ increase of sensitivity between NAAT modalities and C. trachomatis culture (26.5\% sensitivity). In the same males who have sex with males (MSM) demographic, sensitivity of $C$. trachomatis culture from pharyngeal sites was $44.4 \%$. This contrasted with NAAT modalities that reported sensitivity of $\geq 66.7 \%$. Ota et al. [80] reported sensitivity of $C$. trachomatis culture and two NAAT modalities from rectal specimens as being $21.1 \%$ and $94.7 \%$, respectively, from an MSM demographic. The same group reported a significant proportion of pharyngeal detection of C. trachomatis via NAAT in the face of a $0 \%$ culture-positive rate. Using a rotating infected patient status, Bachmann et al. [81] determined C. trachomatis culture sensitivity to be 36.1$45.7 \%$ from rectal swabs in a combined MSM and at-risk female demographic.

Early data suggested PCR utility in the detection of C. trachomatis from ocular specimens $[82,83]$, with one report documenting a $26 \%$ increase in overall $C$. trachomatis detection over that derived from DFA [83]. Commercial PCR additionally proved to have sufficient diagnostic capacity for ocular chlamydia. Kowalski et al. [84] reported $88.1 \%$ sensitivity and $100 \%$ specificity of AMP on adult conjunctival specimens. Hammerschlag et al. [85] documented 92.3\% sensitivity of AMP derived from infant specimens. Studies have also spoken to the utility of nasopharyngeal and nasal discharge specimens in both diagnosis and predictive value of antimicrobial therapy in the context of chlamydial conjunctivitis $[85,86]$. Children with a positive $C$. trachomatis AMP result on a nasal discharge at the commencement of macrolide therapy had an odds ratio of 5.15 to yield a positive AMP result from an ocular specimen two months after therapy when compared to children with a negative AMP result from nasal discharge at baseline [86].

Comparisons of commercial NAAT modalities for the detection of $C$. trachomatis from non-FDA-indicated extragenital sources have ensued. Schachter et al. [76] reported that $32.9 \%$ of C. trachomatis-positive vaginal screening results obtained by AC2 could not be replicated via ProbeTec analysis of a corresponding first-void urine specimen. $64.7 \%$ sensitivity of AMP for the detection of $C$. trachomatis has been demonstrated from rectal specimens when compared to AC2 [80]. The same study reported 33.3\% AMP sensitivity for the detection of pharyngeal $C$. trachomatis using a reference infected patient status in what turned out to be a very low-incidence specimen source. Furthermore, AC2 was $30 \%$ and $33 \%$ more sensitive than ProbeTec in detection of C. trachomatis nucleic acid from rectal and pharyngeal sites, respectively. For diagnosis of rectal chlamydia using a rotating infected patient status, Bachmann et al. [81] calculated sensitivity ranges of AMP, ProbeTec, and AC2 at 80.7$95.5 \%, 92.2-100 \%$, and $100 \%$. Ota et al. [80] reported that AC2 outperformed ProbeTec by $15-20 \%$ in an MSM demographic in terms of sensitivity from rectal and pharyngeal specimens, respectively. In further support of this paradigm, in studies of N. gonorrhoeae detection from pharyngeal and rectal sites, Bachmann and colleagues $[59,81]$ determined the performance of AMP to be inferior to that of ProbeTec or AC2. In a study of an MSM demographic [87], of 86 pharyngeal and 99 rectal specimens that generated a positive AC2 result, only $32.6 \%$ and $34.3 \%$ were positive by $N$. gonorrhoeae culture, respectively. Of the 102 glans specimens positive for $N$. gonorrhoeae by AC2, 96-100\% of these results were confirmed by secondary NAAT. In contrast, a higher percentage of $N$. gonorrhoeae AMP-positive rectal swabs were positive by $N$. gonorrhoeae culture when compared to analogous AC2 data. Collectively, these findings challenge the overall analytical sensitivity of AMP for the detection of STI etiologies from pharyngeal and rectal sources.

Limited comparative data exist on molecular detection of C. trachomatis from ocular specimens. In an Italian study reported by Fontana et al. [88], overall sensitivity of ProbeTec for the detection of $C$. trachomatis was $76.5 \%$ when compared to a laboratory-developed PCR assay targeting $16 \mathrm{~S}$ rDNA which detected all 34 positive specimens. Two of three C. trachomatis-positive ocular specimens were detected by ProbeTec (all three were detected by the assay targeting $16 \mathrm{~S}$ rDNA). It was noted that a second laboratory-developed PCR assay targeting C. trachomatis plasmid DNA detected 28 of 34 overall positive specimens ( 2 of 3 positive ocular specimens). A C. trachomatis plasmid DNA deletion rate of $17.6 \%$ was noted in this study. In a study conducted in Ethiopia, Yang et al. [89] demonstrated that a TMA-based assay specific solely for $C$. trachomatis $16 \mathrm{~S}$ rRNA (ACT; GenProbe) had a detection rate of 59\% which was in contrast to an AMP-derived 28\% detection rate. Increased detection of ocular infection by TMA was independent of active clinical disease $(P \leq 0.004)$. These findings extended those of a previous study [90]. Seven of 15 TMA-positive/AMPnegative specimens had detectable rRNA subsequent to a 1:10 dilution of the original ocular specimen. Taken together, these data are relevant because rRNA detection can mitigate the possibility of $C$. trachomatis plasmid deletion [88], low C. trachomatis burden has been demonstrated in the context of trachoma management $[91,92]$, and rRNA concentration far exceeds that of genomic DNA and plasmid DNA in C. trachomatis [93].

4.4. Trends. Several of the aforementioned findings, plus considerations related to the detection of other sexuallytransmitted agents $[20,94]$, may account for increased utilization of commercial TMA for the detection of C. trachomatis. Surveys of NAAT modalities employed by clinical laboratories in the United States, conducted by the College of American Pathologists laboratory accreditation program [95], have demonstrated an approximate 30\% increase in the utilization of AC2 for C. trachomatis screening from 2003-2010 (Figure 3). Overall participant enrollment in these surveys has ranged from 525 laboratories in early 2003 to an average of 925 in 2010. Furthermore, a 2004 survey of United States public health laboratories reported that $87 \%$ of respondents performed NAAT for the detection of C. trachomatis, while less than $40 \%$ offered nucleic acid 


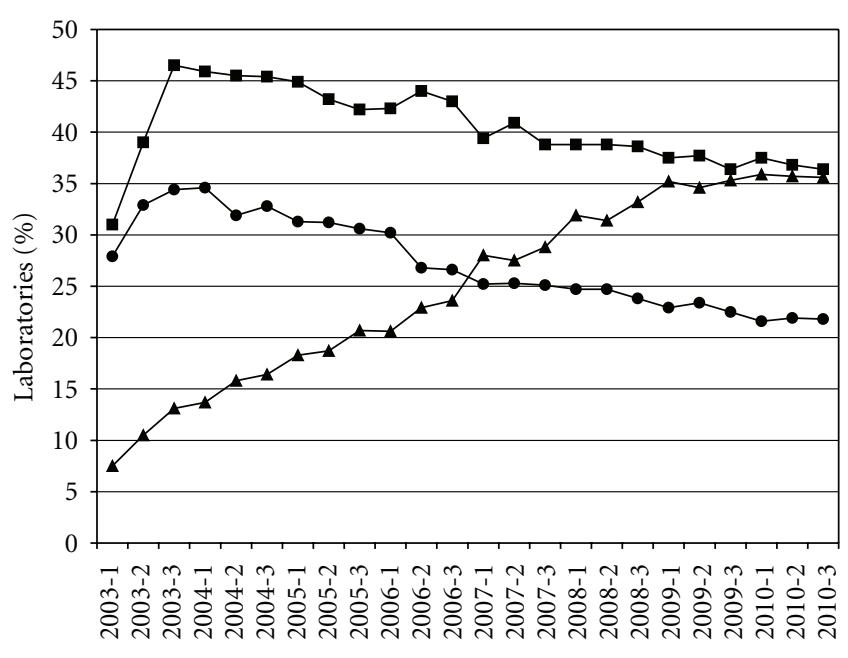

Proficiency challenge

Figure 3: Utilization of AMPLICOR (circles), BD ProbeTec ET (squares), and APTIMA Combo 2 (triangles) platforms for Chlamydia trachomatis screening in United States laboratories, as captured by College of American Pathologists proficiency testing participant summary data collections, 2003-2010. Data are adapted from [95].

hybridization. Of the laboratories that offered NAAT, $50 \%$ utilized ProbeTec, while 48\% performed AC2 [96].

Abbott Laboratories (Des Plaines, Ill, USA) has recently introduced a testing platform $(m 2000)$ to accommodate both automated specimen processing and real-time multiplex PCR for the detection of regions of the C. trachomatis cryptic plasmid and N. gonorrhoeae opacity (Opa) gene. Limit of the detection was reported at 20 copies of DNA for each analyte [97]. When assessed against AMP and ProbeTec using residual genital swab material, the $m 2000$ demonstrated 96.3-99.1\% concordance of positive C. trachomatis result, with $98.2-100 \%$ concordance of negative result. Urine testing demonstrated high concordance with AC2 and ProbeTec for C. trachomatis-negative results $(\geq 98.9 \%)$, with $93.7 \%$ and $96.8 \%$ concordance rates, respectively, for C. trachomatispositive results [97]. Levett et al. [72] compared automated versions of ProbeTec (Viper system) and AC2 (TIGRIS DTS system) to the $m 2000$ for $C$. trachomatis urine testing and reported that sensitivity ranged from 96.9\% $(m 2000)$ to 99.0\% (AC2).

A strain of $C$. trachomatis with a 377-base pair cryptic plasmid deletion [98] is implicated in the purported decreased rates of positive $C$. trachomatis NAAT results reported in clinical laboratories in Sweden beginning around 2004 [99]. Interestingly, Herrmann et al. reported a proportional rate for this C. trachomatis variant ranging from $20 \%$ to $64 \%$ in regions that utilized either $m 2000$ or a commercial real-time PCR system distributed by Roche Molecular Systems. In contrast, in locales that utilized ProbeTec, the proportional rate of the $C$. trachomatis variant ranged from $7 \%$ to $19 \%$ [99]. Despite these Swedish prevalence data, the variant has been identified from clinical specimens in only two neighboring countries [100].
Amidst concern that the $m 2000$ demonstrated poor utility in the detection of European plasmid mutant $C$. trachomatis strains $[99,101]$, modifications were made to the Abbott Laboratories primer sets. This reformulated product (Abbott RealTime CT) was assessed, along with AC2 and version 2 of the COBAS TaqMan CT test (Roche Molecular Systems), against a panel of 148 C. trachomatis-positive urine specimens [102]. Nearly $25 \%$ of these specimens contained the variant $C$. trachomatis strain. Assay specificity was nearly $100 \%$ for all three systems. Sensitivity of the COBAS TaqMan CT test (83.0\%) was outpaced by analogous indices for the Abbott Laboratories reformulation (95.3\%) and AC2 (99.3\%). A separate study [103] reported that the reformulated Abbott Laboratories assay yielded slightly higher sensitivity than ProbeTec.

\section{Addressing the Issue of Specificity: Confirmatory Testing}

5.1. Principle and Methods. Efforts to enhance C. trachomatis NAAT sensitivity theoretically come at the expense of assay specificity. Overall scenarios that could generate such falsepositive results include the nucleic acid target of interest being present within other organisms endogenous to a specimen, the detection system generating signal in the absence of target; iatrogenic contamination, and, clerical errors [29]. In light of this, past literature from the CDC stated that NAAT assays for $C$. trachomatis are indeed screening assays and that an initial positive result should be considered strictly as presumptive evidence of infection. As such, the CDC deemed necessary the verification of a positive screen in cases that could have adverse medical or psychosocial impact [29]. Furthermore, consideration should be given for the verification of positive NAAT screens for analyses performed in low-prevalence STI populations that would render positive predictive values on the order of $90 \%$ or less.

CDC-advocated approaches to additional molecular testing have been four-fold: (1) testing a second primary clinical specimen with an assay that utilizes a different target and a different format, (2) testing the original primary clinical specimen with an assay that utilizes a different target and a different format, (3) repeating the original test on the original primary clinical specimen with a competitive probe, and, (4) repeating the original test on the original specimen. Laboratories that choose commercial nucleic acid hybridization as the method of choice for the detection of $C$. trachomatis can utilize a direct and competitive probe-based nucleic acid hybridization technology $[39,40]$. However, it is not advisable to utilize less-sensitive signal amplification or nucleic acid hybridization technologies to confirm a positive screen derived by NAAT [29]. The method advocated first and foremost may not be a reality in certain healthcare environments or in the public health sector due, in part, to the difficulty in successfully contacting a patient to return for specimen recollection.

5.2. Repeat Testing. Recent literature suggests that the paradigm of repeat testing may introduce difficulties to the 
final interpretation of NAAT results. Culler et al. [104] reported that $5.3 \%$ of initially positive $C$. trachomatis screens yielded by ProbeTec failed to duplicate results upon repeat testing. Castriciano et al. [105] demonstrated that 93.1\% of initially positive $C$. trachomatis screens derived by AMP remained positive upon repeat testing of original lysates. This value dropped to $85.3 \%$ upon testing a second nucleic acid extraction. Schachter et al. [106] reported that only 83.8\% of positive C. trachomatis screens derived by ProbeTec retained positive status upon repeat testing. This value was elevated to $92.5 \%$ upon a second repeat ProbeTec assay. In contrast, $96.7 \%$ and $97.7 \%$ of $\mathrm{AMP}$ and $\mathrm{AC} 2$ screens, respectively, generated a positive result upon repeat testing. This phenomenon has also been noted in molecular detection of $N$. gonorrhoeae. In the study of Culler et al. [104], $10.7 \%$ of positive N. gonorrhoeae screens obtained via ProbeTec failed to retain positive status upon repeat testing. Using multiple specimen sources, Moncada et al. [87] demonstrated that $89.3 \%$ of positive N. gonorrhoeae screens derived by ProbeTec retained positive status by repeat testing. This value increased to $92.4 \%$ and $93.1 \%$ upon a second and third repeat test, respectively. In contrast, repeat testing of positive screens initially derived by AMP and AC2 retained positive status $95.7 \%$ and $96.4 \%$ of the time, respectively.

5.3. Low-Positive Screens. A further delineation of positive NAAT screens reveals an additional conundrum in terms of a role for confirmatory testing in final result interpretation. $80.8 \%$ of low-positive C. trachomatis screens derived from ProbeTec (defined as signal detection method other than acceleration (MOTA) scores from 2000 to 9999) remained positive upon repeat testing, while only $33.3 \%$ of $N$. gonorrhoeae screens retained the positive status [104]. This paradigm may be of greater consideration when studying a highly sensitive assay such as AC2. Two reports $[107,108]$ documented positive status retention rates of $42-63 \%$ for low-positive C. trachomatis screens (defined as relative light unit values between 100,000 and 1,000,000). In contrast, Dunham et al. [107] determined that only $31.6 \%$ of lowpositive N. gonorrhoeae screens retested positive. In a highprevalence population for both C. trachomatis and N. gonorrhoeae, $71.3 \%$ and $58.5 \%$ of low-positive C. trachomatis and $N$. gonorrhoeae screens, respectively, yielded positive results upon repeat testing [109]. No significant difference existed between the percentages of low-positive $C$. trachomatis and $N$. gonorrhoeae screens that remained positive by repeat testing $(P=0.10)$. Despite the fact that repeat testing potentiates result interpretation challenges, this low-positive phenomenon, even under the auspices of $\mathrm{AC} 2$, presents itself in just $2 \%$ of $C$. trachomatis screens and less than $0.1 \%$ of $N$. gonorrhoeae screens performed [107, 109].

5.4. Alternative Target Testing. A fourth CDC-advocated practice, the utilization of an alternative NAAT system or platform, has met with variable success. Schachter et al. [110] reported that while AC2 confirmed $96.9 \%$ of positive ProbeTec screens $(95.4 \%$ in urine specimens, $98.6 \%$ in genital swab specimens), ProbeTec was able to confirm only $82.0 \%$ of positive C. trachomatis screens derived from AC2 $(85.3 \%$ in urine specimens, $78.9 \%$ in genital swab specimens). Chernesky et al. [64] reported $69.6 \%$ and $80.3 \%$ rates of confirmation of positive AC2 urine C. trachomatis screens by AMP and ProbeTec, respectively. Analogous rates for confirmation of positive AC2 endocervical C. trachomatis screens were $62.9 \%$ and $70.9 \%$. In contrast, $98-100 \%$ of positive urine or genital swab C. trachomatis screens yielded by ProbeTec or AMP were confirmed by AC2. In a small subset of specimens that tested equivocal for $C$. trachomatis via AMP or yielded a discrepant result in the context of a combined reference standard, Peterson et al. [111] reported that only $23.1 \%$ of specimens yielded a concordant result when subjected to separate PCR assays targeting different sequences. A C. trachomatis concordance rate of $82.1 \%$ was demonstrated from initial nucleic acid extracts when commercial ligase chain reaction-positive urine screens were tested by AMP [105]. These data further substantiate differences in analytical sensitivity of these NAAT modalities. Similar findings were derived from $N$. gonorrhoeae confirmatory testing. Moncada et al. [87] demonstrated that percentages of positive N. gonorrhoeae screens derived by ProbeTec that were confirmed by AC2 and AMP analysis were $85.0 \%$ and $78.4 \%$, respectively, and that $84.6 \%$ of positive AC2 $N$. gonorrhoeae screens were confirmed by ProbeTec. Yet when similar analysis was restricted to male urine specimens, nearly all positive $N$. gonorrhoeae screens, independent of modality, were confirmed by secondary NAAT.

APTIMA CT and GC assays have allowed for detailed analysis of the CDC-advocated practice of confirmatory testing using an alternative nucleic acid target. Sensitivity and specificity values greater than $96 \%$ were demonstrated for these assays in a multicenter study of MSM using both urethral swabs and urine specimens [112]. Boyadzhyan et al. [32] reported complete concordance of AC2 C. trachomatis and APTIMA CT assay results on 253 urine specimens and 422 genital swab specimens collected from either gender. Schachter and colleagues $[106,110]$ demonstrated that the APTIMA CT assay confirmed $98-99 \%$ of positive AC2 screens, with just slight differences noted between concordance values from urine and genital swab specimens [106]. Comparable data have been reported with respect to N. gonorrhoeae confirmatory testing. Golden et al. [113] reported that 258 of 265 positive N. gonorrhoeae screens of female urine or endocervical specimens derived by AC2 also yielded a positive APTIMA GC assay result. Moncada et al. [87] demonstrated that $95.7 \%$ of combined gender specimens initially screening positive by AC2 yielded a positive APTIMA GC result.

5.5. Comparisons of Repeat Testing to Alternative Target Confirmation. Data from a five-state United States moderateprevalence chlamydia population (cumulative C. trachomatis infection rate of 312.7 per 100,000 population) revealed that repeat testing versus performance of the APTIMA CT assay on AC2-positive C. trachomatis screens demonstrated 95\% concordance of the final result [114]. Schachter et al. [106] utilized a moderate-prevalence California population 
(336.7 cases per 100,000 population) to demonstrate an $84-98 \%$ rate of initial AC2 screen confirmation by repeat testing and a potentially elevated rate (89-99\%) of initially positive screens confirmed by secondary NAAT. In a highprevalence population (684.0 cases per 100,000 population), significantly more low-positive $C$. trachomatis screens were confirmed by the APTIMA CT assay than by duplicate repeat testing [109]. However, these authors noted that utilization of alternative target TMA for confirmation raised overall AC2 C. trachomatis positive predictive value only $1.8 \%$ over that derived from repeat testing. Similar findings characterized $N$. gonorrhoeae screen verification algorithms. Zanto et al. [114] demonstrated 90\% concordance in final $N$. gonorrhoeae AC2 result derived by repeat testing versus alternative target TMA. Moncada et al. [87] summarized their side-by-side comparison of the two advocated methods by noting that $89-96 \%$ of specimens positive by initial NAAT were confirmed by repeat testing and that $85-98 \%$ of initial screening results were confirmed via secondary NAAT. In a high-prevalence gonorrhea population (265.9 cases per 100,000 population), confirmatory testing via the APTIMA GC assay demonstrated only a 5\% increase in the rate of AC2 low-positive result retention [109].

\section{Test of Cure}

Due to very high microbial cure rates exhibited by azithromycin and doxycycline in a recent meta-analysis [115], the CDC does not advocate $C$. trachomatis test-of-cure analysis in males or in nongravid females, unless therapeutic compliance is questioned, symptoms persist, or reinfection is suspected [116]. Workowski et al. [117] utilized an in-house PCR to demonstrate a reduction in rate of endocervical detection of C. trachomatis from 50\% immediately following completion of doxycycline therapy to $15 \%$ seven days later. Nucleic acid was not detected at the two-week interval. Gaydos et al. [118] reported greater C. trachomatis-specific nucleic acid recovery rates for commercial ligase chain reaction (37-73\%, interval dependent) over those of AMP (21-40\% for similar intervals) within the first six days following the completion of therapy.

In contrast, an in-house PCR detected C. trachomatis nucleic acid from $25 \%$ of endocervical swabs collected three weeks after the completion of therapy [119]. In the same study, nucleic acid sequence-based amplification, an RNA amplification technology, yielded a C. trachomatis detection rate of only $6.7 \%$ and $8.0 \%$ from urine and endocervical specimens, respectively, one week after the completion of therapy. Comparator percentages were $26.7 \%$ and $84.0 \%$ for the PCR. Bianchi et al. [120] subjected post treatment urine specimens to AMP and the Gen-Probe first generation TMA assay. Kinetics of both systems was essentially equivalent in females, demonstrating full clearance within six days. Similar results were generated in a smaller sampling of males.

According to recently published CDC recommendations [116], test-of-cure protocols are unnecessary for patients who have completed antimicrobial therapy for $N$. gonorrhoeae infection because multiple lines of therapy have proven efficacious [121]. Exceptions to this paradigm are in the minority and are potentially linked to increasing resistance of $N$. gonorrhoeae to fluoroquinolone agents [122, 123]. However, high prevalence of $N$. gonorrhoeae infection exists in patients who have had gonorrhea in the preceding months [124]. These data imply that the detection of $N$. gonorrhoeae post-treatment may actually be reflective of reinfection rather than treatment failure. If symptoms persist in patients following the completion of therapy, clinicians may consider re-testing patients, via culture modalities, for the ultimate purposes of antimicrobial susceptibility testing. This approach is hypothetically far more efficacious in the management of $N$. gonorrhoeae infection than in $C$. trachomatis infection because of stark differences in culture sensitivity [28].

A paucity of studies has characterized an auxiliary role for NAAT in gonorrhea test-of-cure. For example, Hanks et al. [125] demonstrated that nucleic acid hybridization was unable to generate $N$. gonorrhoeae signal from genital and urine specimens of patients between six and eleven days post-completion of antimicrobial therapy for $N$. gonorrhoeae infection. Bachmann et al. [126], utilizing a commercial ligase chain reaction, reported the median time to a negative $N$. gonorrhoeae urine assay being one day for males and two days for females upon completion of therapy. Among females, the mean clearance time proved greater for genital specimens ( 2.8 days) than for urine specimens ( 1.7 days; $P=$ $0.008)$. An intermittent shedding phenomenon was observed in $15 \%$ of males and $25 \%$ of females during the threeweek follow-up period. Women who shed $N$. gonorrhoeae nucleic acid intermittently were twice as likely to have a genital specimen yield detectable $N$. gonorrhoeae compared to a urine specimen. In one female patient, such detection occurred 19 days after the completion of therapy.

\section{Utility in Medicolegal Settings}

The shorter length of the vagina in prepubescent girls, combined with its columnar epithelial cell lining and alkaline environment, can predispose this population to infection with sexually transmitted agents including C. trachomatis [127]. The extrapolation of $C$. trachomatis detection in children beyond the neonatal period to sexual abuse [116] has some limitations. It has been estimated that $20 \%$ of infants born to women with active $C$. trachomatis infection can acquire the infection in rectal and vaginal sites [128]. Persistence of the organism acquired in perinatal fashion may last 2-3 years [129]. Moreover, retrospective chart reviews [130-133], subcomponents of which utilized C. trachomatis NAAT, have outlined very low incidence of $C$. trachomatis detection (0.5-3.1\%) in the context of child sexual abuse. Schachter et al. [79], within a significant at-risk population for STI acquisition, reported lower $C$. trachomatis detection rates from oropharyngeal and rectal sites $(0.8 \%$ and $6.1 \%$, resp.) than those for $N$. gonorrhoeae. Taken together, it must be noted that the positive predictive value of even very highly specific NAAT screens can be compromised by low disease prevalence in a given setting [134].

Largely as a result, it has been a long-standing axiom that C. trachomatis cultivation has more validity in medicolegal 
TABle 3: Recommended diagnostic modalities and specimen sources for evaluation of child sexual abuse victims. Data are adapted from [116].

\begin{tabular}{lcccc}
\hline Diagnostic modality & & \multicolumn{2}{c}{ Recommended specimen sources for STI etiology } \\
& Female & Chlamydia trachomatis & Neisseria gonorrhoeae \\
& & Male & Female & Male \\
\hline Culture & Anus & Anus & Oropharynx & Anus \\
& & Anus & Urethra \\
& Vagina & Urethral discharge & & Urethral discharge \\
& Urine & & Vagina & \\
NAAT $^{\dagger}$ & Vagina & & Urine & \\
& & & Vagina & \\
\hline
\end{tabular}

${ }^{\dagger}$ Nucleic acid amplification testing.

proceedings than results derived from NAAT [135]. Despite this, in a limited sexual abuse victim dataset, MatthewsGreer et al. [136] described 11 instances of C. trachomatis culture data being corroborated by a positive PCR result. Two rectal specimens were culture positive/PCR negative, while one rectal and three female genital swabs yielded culture-negative/PCR-positive results. Girardet et al. [137] reported that 18 of $215(8.4 \%)$ possible pediatric sexual abuse victims generated a positive NAAT for $C$. trachomatis from a noninvasive urine specimen. Cultures for $C$. trachomatis were positive in only $44 \%$ of instances. Kellogg et al. [138] reported 11-18\% agreement between C. trachomatis culture results and those derived from ligase chain reaction or PCR performance on urine and vaginal specimens collected from girls who reported abusive sexual contact. Among 485 girls being evaluated for sexual abuse, sensitivity of urine C. trachomatis NAAT was $100 \%$ when compared to vaginal culture [139]. Eight additional patients yielded positive urine $C$. trachomatis NAAT in the face of negative culture $(P=0.018)$.

Interestingly, in 2001 Hammerschlag [134] stated that the advancement of NAAT evidence in courts of law was hindered by the paucity of appropriate commercially available confirmatory assays. With the advent of the APTIMA CT assay and defined performance characteristics $[32,112]$, perhaps this scenario warrants additional consideration. CDC recommendations have varied on this topic within the past five years. 2006 recommendations for the management of STI [140] stated that NAAT might be a viable alternative in the detection of $C$. trachomatis if culture systems for the organism are unavailable and if a method of confirmation is available. Noted means of confirmation included secondary FDA-cleared NAAT targeting a different sequence than the primary screening method [140]. This nonculture option for the detection was not advocated for laboratory diagnosis of N. gonorrhoeae. Black et al. [139] remarked that urine NAAT methodologies (with subsequent confirmatory testing) are adequate as a new forensic standard in children suspected of being sexual abuse victims. The recently published guidelines [116] place primary focus on standardized anal (both genders), urethral discharge (males), and vaginal culture techniques for both $C$. trachomatis and $N$. gonorrhoeae (Table 3). The paucity of specimen source options available for C. trachomatis culture is related to low source-specific organism prevalence rates and the paradigm of chlamydial persistence following perinatal acquisition. NAAT for $C$. trachomatis and $N$. gonorrhoeae from vaginal and urine specimens is recommended in girls as an alternative to culture. Furthermore, in the context of sexual assault in adults and adolescents, the latest recommendations call for the performance of FDA-cleared NAAT for either $C$. trachomatis or $N$. gonorrhoeae upon initial examination. The topic of STI detection in the context of medicolegal testing has been reviewed extensively by Hammerschlag and Guillén [141].

\section{Conclusions}

Independent of specimen transport conditions, sensitivity of C. trachomatis culture is greatly inferior to those of amplified molecular methods that have since largely replaced signal amplification and nucleic acid hybridization assays. Because molecular-based testing for $N$. gonorrhoeae is simultaneously provided within commercial molecular assays for C. trachomatis, many laboratories subsequently forego sole reliance upon culture methods for $N$. gonorrhoeae detection from urine and genital sources. Sensitivity differences between commercial PCR, SDA, and TMA have been delineated in the literature, both in clinical and in vitro settings. The aforementioned specimen types are applicable to $\mathrm{AC} 2$, with the addition of vaginal swab and gynecological specimens (Table 4). A limited role for $C$. trachomatis culture may be seen in medicolegal settings or for cultivation from specimen sources that are not indicated for FDA-approved NAAT. However, studies have emerged advocating a highly sensitive NAAT modality, such as commercial TMA, to augment culture methodology for accurate detection of $C$. trachomatis from extragenital sites.

Validity of results generated by highly sensitive modalities has been addressed with a variety of confirmatory testing algorithms. Limitations to follow-up testing include clinicians not routinely providing two specimens for evaluation and the prohibitive expense for some laboratories to either modify an existing molecular assay to target a different nucleic acid sequence or validate secondary NAAT. Even when NAAT is utilized as a means of confirmation, 
TABLE 4: United states food and drug administration-approved specimen sources for commercially available Chlamydia trachomatis nucleic acid amplification testing. Data are derived from [50-52].

\begin{tabular}{|c|c|c|c|c|}
\hline Modality & Symptomatic female & Symptomatic male & Asymptomatic female & Asymptomatic male \\
\hline \multirow{2}{*}{ AMPLICOR } & Endocervix & Urethra & Endocervix & Urethra \\
\hline & Urine & Urine & Urine & Urine \\
\hline \multirow{2}{*}{ BD ProbeTec ET } & Endocervix & Urethra & Endocervix & Urethra \\
\hline & Urine & Urine & Urine & Urine \\
\hline \multirow{5}{*}{ APTIMA Combo 2} & Endocervix & Urethra & Endocervix & Urethra \\
\hline & Urine & Urine & Urine & Urine \\
\hline & Vagina $^{\mathrm{a}}$ & & Vagina $^{\mathrm{a}}$ & \\
\hline & Gynecology Exam ${ }^{\mathrm{b}}$ & & Gynecology Exam ${ }^{\mathrm{b}}$ & \\
\hline & & & Vagina $^{c}$ & \\
\hline
\end{tabular}

${ }^{\mathrm{a}}$ Clinician collected.

${ }^{b}$ Collected in PreservCyt Solution; processed with ThinPrep 2000 system (Cytyc Corporation, Marlborough, Mass, USA).

'Patient collected.

differences in performance characteristics of these assays, deficiencies in result reproducibility for a given specimen using the same testing modality, and potential differences in sensitivity related to heterologous specimen collection media/transport devices have been reported [106]. Even when repeat testing is factored into this paradigm, additional generated data may be difficult to interpret, especially when considering the extremely high rates of sensitivity and specificity already inherent to these screening assays. It must be kept in mind that CDC recommendations related to $C$. trachomatis screening diagnostics have not been updated since 2002 [29]. On the basis of the discussion provided in Section 5 of this review, any subsequent revision may result in significant changes related to this paradigm.

While currently not widely accepted as medicolegal evidence due to concerns over specificity, admission of NAAT results may eventually become standard practice in courts of law. Prominent acceptance of alternative target confirmatory testing may have to play a significant role for this to occur, particularly with a highly sensitive method such as commercial TMA. Viable test-of-cure options are not extensive in the setting of chlamydia due to meager sensitivity of $C$. trachomatis culture. At the same time, amplified molecular test-of-cure protocols are deemed unnecessary in a majority of settings due to efficacious therapeutic regimens. Auxiliary studies utilizing NAAT demonstrate microbiological cure approximately 7-14 days after therapy, yet investigations in this vein using latest-generation commercial TMA would be compelling.

Chlamydia prevalence has experienced a significant upswing in the United States over the past 15 years. Clinical presentation of male urethritis exhibits overlap with that of nongonococcal urethritis. In females, symptoms of chlamydia can resemble those of gonorrhea or trichomoniasis. These data predicate the importance of laboratory detection of C. trachomatis. Poor C. trachomatis culture sensitivity signifies the importance of nonculture diagnostic modalities. With respect to the utilization of the rapid, nonamplification methods for $C$. trachomatis detection, one must be cautious to the actual "point-of-care" benefit therein, as many studies have proven these methods to have much lower analytical sensitivity than NAAT. Consequently, as nucleic acid-based diagnostic assays continue to improve, a greater presence for such testing needs to be established in both small- and largescale clinical laboratory settings.

\section{References}

[1] Centers for Disease Control and Prevention, "Sexually transmitted disease surveillance, 2010,” Tech. Rep., Department of Health and Human Services, Atlanta, Ga, USA, 2009.

[2] W. Cates Jr. and J. N. Wasserheit, "Genital chlamydial infections: epidemiology and reproductive sequelae," American Journal of Obstetrics and Gynecology, vol. 164, no. 6, pp. 17711781, 1991.

[3] J. K. Podgore, K. K. Holmes, and E. R. Alexander, "Asymptomatic urethral infections due to Chlamydia trachomatis in male U.S. military personnel," Journal of Infectious Diseases, vol. 146, no. 6, p. 828, 1982.

[4] G. H. Karam, D. H. Martin, and T. R. Flotte, "Asymptomatic Chlamydia trachomatis infections among sexually active men," Journal of Infectious Diseases, vol. 154, no. 5, pp. 900903, 1986.

[5] E. W. Hook III, C. A. Reichart, D. M. Upchurch, P. Ray, D. Celentano, and T. C. Quinn, "Comparative behavioral epidemiology of gonococcal and chlamydial infections among patients attending a Baltimore, Maryland, sexually transmitted disease clinic," American Journal of Epidemiology, vol. 136, no. 6, pp. 662-672, 1992.

[6] E. Lycke, G.-B. Löwhagen, G. Hallhagen, G. Johannisson, and K. Ramstedt, "The risk of transmission of genital Chlamydia trachomatis infection is less than that of genital Neisseria gonorrhoeae infection," Sexually Transmitted Diseases, vol. 7, no. 1, pp. 6-10, 1980.

[7] B. P. Katz, V. A. Caine, and R. B. Jones, "Estimation of transmission probabilities for chlamydial infection," in Chlamydial Infections, W. R. Bowie, H. D. Caldwell, R. P. Jones et al., Eds., pp. 567-570, Cambridge University Press, Cambridge, UK, 1990.

[8] T. C. Quinn, C. Gaydos, M. Shepherd et al., "Epidemiologic and microbiologic correlates of Chlamydia trachomatis infection in sexual partnerships," The Journal of the American Medical Association, vol. 276, no. 21, pp. 1737-1742, 1996. 
[9] R. R. Hooper, G. H. Reynolds, O. G. Jones et al., "Cohort study of venereal disease. I: the risk of gonorrhea transmission from infected women to men," American Journal of Epidemiology, vol. 108, no. 2, pp. 136-144, 1978.

[10] J. S. L. Lin, S. P. Donegan, T. C. Heeren et al., "Transmission of Chlamydia trachomatis and Neisseria gonorrhoeae among men with urethritis and their female sex partners," Journal of Infectious Diseases, vol. 178, no. 6, pp. 1707-1712, 1998.

[11] W. E. Stamm, R. B. Jones, and B. E. Batteiger, "Chlamydia trachomatis (trachoma, perinatal infections, lymphogranuloma venereum, and other genital infections)," in Principles and Practice of Infectious Diseases, G. L. Mandell, J. E. Bennett, and R. Dolin, Eds., pp. 2239-2255, Elsevier, Philadelphia, Pa, USA, 6th edition, 2005.

[12] M. D. Nettleman, R. B. Jones, S. D. Roberts et al., "Costeffectiveness of culturing for Chlamydia trachomatis: a study in a clinic for sexually transmitted diseases," Annals of Internal Medicine, vol. 105, no. 2, pp. 189-196, 1986.

[13] W. E. Stamm, L. A. Koutsky, J. K. Benedetti, J. L. Jourden, R. C. Brunham, and K. K. Holmes, "Chlamydia trachomatis urethral infections in man. Prevalence, risk factors, and clinical manifestations," Annals of Internal Medicine, vol. 100, no. 1, pp. 47-51, 1984.

[14] J. A. McCutchan, "Epidemiology of venereal urethritis: comparison of gonorrhea and nongonococcal urethritis," Reviews of Infectious Diseases, vol. 6, no. 5, pp. 669-688, 1984.

[15] W. O. Harrison, R. R. Hooper, P. J. Wiesner, A. F. Campbell, W. W. Karney, and G. H. Reynolds, "A trial of minocycline given after exposure to prevent gonorrhea," The New England Journal of Medicine, vol. 300, no. 19, pp. 1074-1078, 1979.

[16] N. F. Jacobs and S. J. Kraus, "Gonococcal and nongonococcal urethritis in men. Clinical and laboratory differentiation," Annals of Internal Medicine, vol. 82, no. 1, pp. 7-12, 1975.

[17] R. Rothenberg and F. N. Judson, "The clinical diagnosis of urethral discharge," Sexually Transmitted Diseases, vol. 10, no. 1, pp. 24-28, 1983.

[18] J. Volk and S. J. Kraus, "Nongonococcal urethritis: a venereal disease as prevalent as epidemic gonorrhea," Archives of Internal Medicine, vol. 134, no. 3, pp. 511-514, 1974.

[19] A. E. Washington, S. Gove, J. Schachter, and R. L. Sweet, "Oral contraceptives, Chlamydia trachomatis infection, and pelvic inflammatory disease," The Journal of the American Medical Association, vol. 253, no. 15, pp. 2246-2250, 1985.

[20] E. Munson, M. Napierala, R. Olson et al., "Impact of Trichomonas vaginalis transcription-mediated amplificationbased analyte-specific-reagent testing in a metropolitan setting of high sexually transmitted disease prevalence," Journal of Clinical Microbiology, vol. 46, no. 10, pp. 3368-3374, 2008.

[21] C. A. Gaydos, T. C. Quinn, D. Willis et al., "Performance of the APTIMA Combo 2 assay for detection of Chlamydia trachomatis and Neisseria gonorrhoeae in female urine and endocervical swab specimens," Journal of Clinical Microbiology, vol. 41, no. 1, pp. 304-309, 2003.

[22] E. Munson and M. A. Firmani, "Molecular diagnosis of Neisseria gonorrhoeae infection in the United States," Expert Opinion in Medical Diagnostics, vol. 3, no. 3, pp. 327-343, 2009.

[23] M. Laga, A. Manoka, M. Kivuvu et al., "Non-ulcerative sexually transmitted diseases as risk factors for HIV-1 transmission in women: results from a cohort study," AIDS, vol. 7, no. 1, pp. 95-102, 1993.
[24] Centers for Disease Control and Prevention, "HIV prevention through early detection and treatment of other sexually transmitted disease-United States recommendations of the advisory committee for HIV and STD prevention," Morbidity and Mortality Weekly Report, vol. 47, no. RR-12, pp. 1-24, 1998.

[25] G. R. Swain, R. A. McDonald, J. R. Pfister, M. S. Gradus, G. V. Sedmak, and A. Singh, "Decision analysis: point-of-care Chlamydia testing vs. laboratory-based methods," Clinical Medicine \& Research, vol. 2, no. 1, pp. 29-35, 2004.

[26] M. S. Pate, P. B. Dixon, K. Hardy, M. Crosby, and E. W. Hook III, "Evaluation of the Biostar Chlamydia OIA assay with specimens from women attending a sexually transmitted disease clinic," Journal of Clinical Microbiology, vol. 36, no. 8, pp. 2183-2186, 1998.

[27] C. I. Bandea, E. H. Koumans, M. K. Sawyer et al., "Evaluation of the rapid BioStar optical immunoassay for detection of Chlamydia trachomatis in adolescent women," Journal of Clinical Microbiology, vol. 47, no. 1, pp. 215-216, 2009.

[28] C. H. Livengood III and J. W. Wrenn, "Evaluation of COBAS AMPLICOR (Roche): accuracy in detection of Chlamydia trachomatis and Neisseria gonorrhoeae by coamplification of endocervical specimens," Journal of Clinical Microbiology, vol. 39, no. 8, pp. 2928-2932, 2001.

[29] Centers for Disease Control and Prevention, "Screening tests to detect Chlamydia trachomatis and Neisseria gonorrhoeae infections-2002," Morbidity and Mortality Weekly Report, vol. 51, no. RR-15, pp. 1-40, 2002.

[30] E. W. Hook III, C. Spitters, C. A. Reichart, T. M. Neumann, and T. C. Quinn, "Use of cell culture and a rapid diagnostic assay for Chlamydia trachomatis screening," The Journal of the American Medical Association, vol. 272, no. 11, pp. 867-870, 1994.

[31] J. R. Schwebke, R. Sadler, J. M. Sutton, and E. W. Hook III, "Positive screening tests for gonorrhea and chlamydial infection fail to lead consistently to treatment of patients attending a sexually transmitted disease clinic," Sexually Transmitted Diseases, vol. 24, no. 4, pp. 181-184, 1997.

[32] B. Boyadzhyan, T. Yashina, J. H. Yatabe, M. Patnaik, and C. S. Hill, "Comparison of the APTIMA CT and GC assays with the APTIMA Combo 2 assay, the abbott LCx assay, and direct fluorescent-antibody and culture assays for detection of Chlamydia trachomatis and Neisseria gonorrhoeae," Journal of Clinical Microbiology, vol. 42, no. 7, pp. 3089-3093, 2004.

[33] C. M. Black, J. Marrazzo, R. E. Johnson et al., "Head-tohead multicenter comparison of DNA probe and nucleic acid amplification tests for Chlamydia trachomatis infection in women performed with an improved reference standard," Journal of Clinical Microbiology, vol. 40, no. 10, pp. 37573763, 2002.

[34] L. van Dommelen, F. H. van Tiel, S. Ouburg et al., "Alarmingly poor performance in Chlamydia trachomatis point-ofcare testing," Sexually Transmitted Infections, vol. 86, no. 5, pp. 355-359, 2010.

[35] L. Greer and G. D. Wendel Jr., "Rapid diagnostic methods in sexually transmitted infections," Infectious Disease Clinics of North America, vol. 22, no. 4, pp. 601-617, 2008.

[36] F. S. Nolte and A. Caliendo, "Molecular detection and identification of microorganisms," in Manual of Clinical Microbiol$\circ g y$, P. R. Murray, E. J. Baron, J. H. Jorgensen, M. L. Landry, and M. A. Pfaller, Eds., pp. 218-244, ASM Press, Washington, DC, USA, 9th edition, 2007. 
[37] W. LeBar, B. Herschman, C. Jemal, and J. Pierzchala, "Comparison of DNA probe, monoclonal antibody enzyme immunoassay, and cell culture for the detection of Chlamydia trachomatis," Journal of Clinical Microbiology, vol. 27, no. 5, pp. 826-828, 1989.

[38] L. M. Clarke, M. F. Sierra, B. J. Daidone, N. Lopez, J. M. Covino, and W. M. McCormack, "Comparison of the Syva MicroTrak enzyme immunoassay and Gen-Probe PACE 2 with cell culture for diagnosis of cervical Chlamydia trachomatis infection in a high-prevalence female population," Journal of Clinical Microbiology, vol. 31, no. 4, pp. 968-971, 1993.

[39] J. A. J. W. Kluytmans, W. H. F. Goessens, J. H. van RijsoortVos, H. G. M. Niesters, and E. Stolz, "Improved performance of PACE 2 with modified collection system in combination with probe competition assay for detection of Chlamydia trachomatis in urethral specimens from males," Journal of Clinical Microbiology, vol. 32, no. 2, pp. 568-570, 1994.

[40] R. J. Limberger, R. Biega, A. Evancoe, L. McCarthy, L. Slivienski, and M. Kirkwood, "Evaluation of culture and the Gen-Probe PACE 2 assay for detection of Neisseria gonorrhoeae and Chlamydia trachomatis in endocervical specimens transported to a state health laboratory," Journal of Clinical Microbiology, vol. 30, no. 5, pp. 1162-1166, 1992.

[41] Y. M. Hale, M. E. Melton, J. S. Lewis, and D. E. Willis, "Evaluation of the PACE 2 Neisseria gonorrhoeae assay by three public health laboratories," Journal of Clinical Microbiology, vol. 31, no. 2, pp. 451-453, 1993.

[42] J. Schachter, E. W. Hook III, W. M. McCormack et al., "Ability of the digene hybrid capture II test to identify Chlamydia trachomatis and Neisseria gonorrhoeae in cervical specimens," Journal of Clinical Microbiology, vol. 37, no. 11, pp. 36683671, 1999.

[43] L. H. Darwin, A. P. Cullen, P. M. Arthur et al., "Comparison of digene hybrid capture 2 and conventional culture for detection of Chlamydia trachomatis and Neisseria gonorrhoeae in cervical specimens," Journal of Clinical Microbiology, vol. 40, no. 2, pp. 641-644, 2002.

[44] K. J. Modarress, A. P. Cullen, W. J. Jaffurs Sr. et al., "Detection of Chlamydia trachomatis and Neisseria gonorrhoeae in swab specimens by the hybrid capture II and PACE 2 nucleic acid probe tests," Sexually Transmitted Diseases, vol. 26, no. 5, pp. 303-308, 1999.

[45] R. K. Saiki, S. Scharf, F. Faloona et al., "Enzymatic amplification of beta-globin genomic sequences and restriction site analysis for diagnosis of sickle cell anemia," Science, vol. 230, no. 4732, pp. 1350-1354, 1985.

[46] R. K. Saiki, D. H. Gelfand, S. Stoffel et al., "Primer-directed enzymatic amplification of DNA with a thermostable DNA polymerase," Science, vol. 239, no. 4839, pp. 487-491, 1988.

[47] B. van der Pol, T. C. Quinn, C. A. Gaydos et al., "Multicenter evaluation of the AMPLICOR and automated COBAS AMPLICOR CT/NG tests for detection of Chlamydia trachomatis," Journal of Clinical Microbiology, vol. 38, no. 3, pp. 1105-1112, 2000.

[48] G. J. J. van Doornum, L. M. Schouls, A. Pijl, I. Cairo, M. Buimer, and S. Bruisten, "Comparison between the LCx probe system and the COBAS AMPLICOR system for detection of Chlamydia trachomatis and Neisseria gonorrhoeae infections in patients attending a clinic for treatment of sexually transmitted diseases in Amsterdam, the Netherlands," Journal of Clinical Microbiology, vol. 39, no. 3, pp. 829-835, 2001.
[49] D. H. Martin, C. Cammarata, B. van der Pol et al., "Multicenter evaluation of AMPLICOR and automated COBAS AMPLICOR CT/NG tests for Neisseria gonorrhoeae," Journal of Clinical Microbiology, vol. 38, no. 10, pp. 3544-3549, 2000.

[50] AMPLICOR ${ }^{\circledR}$ CT/NG Test for Chlamydia Trachomatis Product Insert, Roche Molecular Systems, Branchburg, NJ, USA, 2003.

[51] BD ProbeTec ${ }^{T M}$ ET Chlamydia trachomatis and Neisseria gonorrhoeae Amplified DNA Assays Product Insert, Becton, Dickinson and Company, Sparks, Md, USA, 2008.

[52] APTIMA COMBO $2{ }^{\circledR}$ Assay Product Insert, Gen-Probe, Incorporated, San Diego, Calif, USA, 2009.

[53] M. C. Little, J. Andrews, R. Moore et al., "Strand displacement amplification and homogeneous real-time detection incorporated in a second-generation DNA probe system, BDProbeTecET," Clinical Chemistry, vol. 45, no. 6 I, pp. 777784, 1999.

[54] B. van der Pol, D. V. Ferrero, L. Buck-Barrington et al., "Multicenter evaluation of the BDProbeTec ET System for detection of Chlamydia trachomatis and Neisseria gonorrhoeae in urine specimens, female endocervical swabs, and male urethral swabs," Journal of Clinical Microbiology, vol. 39, no. 3, pp. 1008-1016, 2001.

[55] J. C. Guatelli, K. M. Whitfield, D. Y. Kwoh, K. J. Barringer, D. D. Richman, and T. R. Gingeras, "Isothermal, in vitro amplification of nucleic acids by a multienzyme reaction modeled after retroviral replication," Proceedings of the National Academy of Sciences of the United States of America, vol. 87, no. 5, pp. 1874-1878, 1990.

[56] J. Compton, "Nucleic acid sequence-based amplification," Nature, vol. 350, no. 6313, pp. 91-92, 1991.

[57] D. J. Farrell, "Evaluation of AMPLICOR Neisseria gonorrhoeae PCR using $\angle P p B$ nested PCR and 16S rRNA PCR," Journal of Clinical Microbiology, vol. 37, no. 2, pp. 386-390, 1999.

[58] B. van der Pol, D. H. Martin, J. Schachter et al., "Enhancing the specificity of the COBAS AMPLICOR CT/NG test for Neisseria gonorrhoeae by retesting specimens with equivocal results," Journal of Clinical Microbiology, vol. 39, no. 9, pp. 3092-3098, 2001.

[59] L. H. Bachmann, R. E. Johnson, H. Cheng, L. E. Markowitz, J. R. Papp, and E. W. Hook III, "Nucleic acid amplification tests for diagnosis of Neisseria gonorrhoeae oropharyngeal infections," Journal of Clinical Microbiology, vol. 47, no. 4, pp. 902-907, 2009.

[60] Z. Nagasawa, Y. Ikeda-Dantsuji, T. Niwa, H. Miyakoshi, and A. Nagayama, "Evaluation of APTIMA Combo 2 for crossreactivity with oropharyngeal Neisseria species and other microorganisms," Clinica Chimica Acta, vol. 411, no. 9-10, pp. 776-778, 2010.

[61] P. Lowe, P. O'Loughlin, K. Evans, M. White, P. B. Bartley, and R. Vohra, "Comparison of the Gen-Probe APTIMA Combo 2 assay to the AMPLICOR CT/NG assay for detection of Chlamydia trachomatis and Neisseria gonorrhoeae in urine samples from Australian men and women," Journal of Clinical Microbiology, vol. 44, no. 7, pp. 2619-2621, 2006.

[62] L. Comanor, F. Anderson, M. Ghany et al., "Transcriptionmediated amplification is more sensitive than conventional PCR-based assays for detecting residual serum HCV RNA at end of treatment," American Journal of Gastroenterology, vol. 96, no. 10, pp. 2968-2972, 2001.

[63] C. Sarrazin, G. Teuber, R. Kokka, H. Rabenau, and S. Zeuzem, "Detection of residual hepatitis C virus RNA by transcription-mediated amplification in patients with 
complete virologic response according to polymerase chain reaction-based assays," Hepatology, vol. 32, no. 4, pp. 818-823, 2000.

[64] M. Chernesky, D. Jang, K. Luinstra et al., "High analytical sensitivity and low rates of inhibition may contribute to detection of Chlamydia trachomatis in significantly more women by the APTIMA Combo 2 assay," Journal of Clinical Microbiology, vol. 44, no. 2, pp. 400-405, 2006.

[65] Y. Ikeda-Dantsuji, I. Konomi, and A. Nagayama, "In vitro assessment of the APTIMA Combo 2 assay for the detection of Chlamydia trachomatis using highly purified elementary bodies," Journal of Medical Microbiology, vol. 54, part 4, pp. 357-360, 2005.

[66] B. J. Wood, P. Rizzo-Price, J. Holden, A. Hardick, T. C. Quinn, and C. A. Gaydos, "The microbicide tenofovir does not inhibit nucleic acid amplification tests for detection of Chlamydia trachomatis and Neisseria gonorrhoeae in urine samples," Journal of Clinical Microbiology, vol. 46, no. 2, pp. 763-765, 2008.

[67] J. Mahony, S. Chong, D. Jang et al., "Urine specimens from pregnant and nonpregnant women inhibitory to amplification of Chlamydia trachomatis nucleic acid by PCR, ligase chain reaction, and transcription-mediated amplification: identification of urinary substances associated with inhibition and removal of inhibitory activity," Journal of Clinical Microbiology, vol. 36, no. 11, pp. 3122-3126, 1998.

[68] R. P. Verkooyen, A. Luijendijk, W. M. Huisman et al., "Detection of PCR inhibitors in cervical specimens by using the AMPLICOR Chlamydia trachomatis assay," Journal of Clinical Microbiology, vol. 34, no. 12, pp. 3072-3074, 1996.

[69] M. Rosenstraus, Z. Wang, S.-Y. Chang, D. DeBonville, and J. P. Spadoro, "An internal control for routine diagnostic PCR: design, properties, and effect on clinical performance," Journal of Clinical Microbiology, vol. 36, no. 1, pp. 191-197, 1998.

[70] C. A. Gaydos, M. Theodore, N. Dalesio, B. J. Wood, and T. C. Quinn, "Comparison of three nucleic acid amplification tests for detection of Chlamydia trachomatis in urine specimens," Journal of Clinical Microbiology, vol. 42, no. 7, pp. 3041-3045, 2004.

[71] J. M. Harris, T. Brink, and C. Welborn, "Performance of the BD ProbeTec ${ }^{T M}$ urine preservative transport with second generation assays for the detection of Chlamydia trachomatis and Neisseria gonorrhoeae on the BD ProbeTec ${ }^{T M}$ ET system," in Proceedings of the 105th General Meeting of the American Society for Microbiology, Atlanta, Ga, USA, June 2005.

[72] P. N. Levett, K. Brandt, K. Olenius, C. Brown, K. Montgomery, and G. B. Horsman, "Evaluation of three automated nucleic acid amplification systems for detection of Chlamydia trachomatis and Neisseria gonorrhoeae in first-void urine specimens," Journal of Clinical Microbiology, vol. 46, no. 6, pp. 2109-2111, 2008.

[73] W. E. Stamm, R. B. Jones, and B. E. Batteiger, "Introduction to chlamydial diseases," in Principles and Practice of Infectious Diseases, G. L. Mandell, J. E. Bennett, and R. Dolin, Eds., pp. 2236-2239, Elsevier, Philadelphia, Pa, USA, 6th edition, 2005.

[74] H. H. Handsfield and P. F. Sparling, "Neisseria gonorrhoeae," in Principles and Practice of Infectious Diseases, G. L. Mandell, J. E. Bennett, and R. Dolin, Eds., pp. 2514-2529, Elsevier, Philadelphia, Pa, USA, 6th edition, 2005.

[75] J. Schachter, W. M. McCormack, M. A. Chernesky et al., "Vaginal swabs are appropriate specimens for diagnosis of genital tract infection with Chlamydia trachomatis," Journal of Clinical Microbiology, vol. 41, no. 8, pp. 3784-3789, 2003.
[76] J. Schachter, M. A. Chernesky, D. E. Willis et al., "Vaginal swabs are the specimens of choice when screening for Chlamydia trachomatis and Neisseria gonorrhoeae: results from a multicenter evaluation of the APTIMA assays for both infections," Sexually Transmitted Diseases, vol. 32, no. 12, pp. 725-728, 2005.

[77] C. A. Gaydos, K. A. Crotchfelt, N. Shah et al., "Evaluation of dry and wet transported intravaginal swabs in detection of Chlamydia trachomatis and Neisseria gonorrhoeae infections in female soldiers by PCR," Journal of Clinical Microbiology, vol. 40, no. 3, pp. 758-761, 2002.

[78] L. A. Cosentino, D. V. Landers, and S. L. Hillier, "Detection of Chlamydia trachomatis and Neisseria gonorrhoeae by strand displacement amplification and relevance of the amplification control for use with vaginal swab specimens," Journal of Clinical Microbiology, vol. 41, no. 8, pp. 3592-3596, 2003.

[79] J. Schachter, J. Moncada, S. Liska, C. Shayevich, and J. D. Klausner, "Nucleic acid amplification tests in the diagnosis of chlamydial and gonococcal infections of the oropharynx and rectum in men who have sex with men," Sexually Transmitted Diseases, vol. 35, no. 7, pp. 637-642, 2008.

[80] K. V. Ota, I. E. Tamari, M. Smieja et al., "Detection of Neisseria gonorrhoeae and Chlamydia trachomatis in pharyngeal and rectal specimens using the BD Probetec ET system, the Gen-Probe Aptima Combo 2 assay and culture," Sexually Transmitted Infections, vol. 85, no. 3, pp. 182-186, 2009.

[81] L. H. Bachmann, R. E. Johnson, H. Cheng et al., "Nucleic acid amplification tests for diagnosis of Neisseria gonorrhoeae and Chlamydia trachomatis rectal infections," Journal of Clinical Microbiology, vol. 48, no. 5, pp. 1827-1832, 2010.

[82] D. Dean, C. R. Pant, and P. O’Hanley, “Improved sensitivity of a modified polymerase chain reaction amplified DNA probe in comparison with serial tissue culture passage for detection of Chlamydia trachomatis in conjunctival specimens from Nepal," Diagnostic Microbiology and Infectious Disease, vol. 12, no. 2, pp. 133-137, 1989.

[83] L. Bobo, B. Munoz, R. Viscidi, T. Quinn, H. Mkocha, and S. West, "Diagnosis of Chlamydia trachomatis eye infection in Tanzania by polymerase chain reaction/enzyme immunoassay," The Lancet, vol. 338, no. 8771, pp. 847-850, 1991.

[84] R. P. Kowalski, M. Uhrin, L. M. Karenchak, R. L. Sweet, and Y. J. Gordon, "Evaluation of the polymerase chain reaction test for detecting chlamydial DNA in adult chlamydial conjunctivitis," Ophthalmology, vol. 102, no. 7, pp. 1016-1019, 1995.

[85] M. R. Hammerschlag, P. M. Roblin, M. Gelling, N. Tsumura, J. E. Jule, and A. Kutlin, "Use of polymerase chain reaction for the detection of Chlamydia trachomatis in ocular and nasopharyngeal specimens from infants with conjunctivitis," Pediatric Infectious Disease Journal, vol. 16, no. 3, pp. 293-297, 1997.

[86] E. W. Gower, A. W. Solomon, M. J. Burton et al., "Chlamydial positivity of nasal discharge at baseline is associated with ocular chlamydial positivity 2 months following azithromycin treatment," Investigative Ophthalmology and Visual Science, vol. 47, no. 11, pp. 4767-4771, 2006.

[87] J. Moncada, E. Donegan, and J. Schachter, "Evaluation of CDC-recommended approaches for confirmatory testing of positive Neissena gonorrhoeae nucleic acid amplification test results," Journal of Clinical Microbiology, vol. 46, no. 5, pp. 1614-1619, 2008. 
[88] C. Fontana, M. Favaro, O. Cicchetti, S. Minelli, E. S. Pistoia, and C. Favalli, "Performance of strand displacement amplification assay in the detection of Chlamydia trachomatis and Neisseria gonorrhoeae," Japanese Journal of Infectious Diseases, vol. 58, no. 5, pp. 283-288, 2005.

[89] J. L. Yang, K. C. Hong, J. Schachter et al., "Detection of Chlamydia trachomatis ocular infection in trachomaendemic communities by rRNA amplification," Investigative Ophthalmology and Visual Science, vol. 50, no. 1, pp. 90-94, 2009.

[90] J. L. Yang, J. Schachter, J. Moncada et al., "Comparison of an rRNA-based and DNA-based nucleic acid amplification test for the detection of Chlamydia trachomatis in trachoma," British Journal of Ophthalmology, vol. 91, no. 3, pp. 293-295, 2007.

[91] N. D. E. Alexander, A. W. Solomon, M. J. Holland et al., "An index of community ocular Chlamydia trachomatis load for control of trachoma," Transactions of the Royal Society of Tropical Medicine and Hygiene, vol. 99, no. 3, pp. 175-177, 2005.

[92] E. S. West, B. Munoz, H. Mkocha et al., "Mass treatment and the effect on the load of Chlamydia trachomatis infection in a trachoma-hyperendemic community," Investigative Ophthalmology and Visual Science, vol. 46, no. 1, pp. 83-87, 2005.

[93] J. W. Mouton, R. Verkooyen, W. I. van der Meijden et al., "Detection of Chlamydia trachomatis in male and female urine specimens by using the amplified Chlamydia trachomatis test," Journal of Clinical Microbiology, vol. 35, no. 6, pp. 1369-1372, 1997.

[94] A. Hardick, J. Hardick, B. J. Wood, and C. Gaydos, "Comparison between the Gen-Probe transcription-mediated amplification Trichomonas vaginalis research assay and realtime PCR for Trichomonas vaginalis detection using a Roche LightCycler instrument with female self-obtained vaginal swab samples and male urine samples," Journal of Clinical Microbiology, vol. 44, no. 11, pp. 4197-4199, 2006.

[95] College of American Pathologists, C. Trachomatis and N. Gonorrhoeae by Nucleic Acid Amplification Participant Summary, HC6A-HC6C, College of American Pathologists, Northfield, Ill, USA, 2003-2010.

[96] L. W. Dicker, D. J. Mosure, R. Steece, and K. M. Stone, "Testing for sexually transmitted diseases in U.S. public health laboratories in 2004," Sexually Transmitted Diseases, vol. 34, no. 1, pp. 41-46, 2007.

[97] R. Marshall, M. Chernesky, D. Jang et al., "Characteristics of the $m 2000$ automated sample preparation and multiplex real-time PCR system for detection of Chlamydia trachomatis and Neisseria gonorrhoeae," Journal of Clinical Microbiology, vol. 45, no. 3, pp. 747-751, 2007.

[98] T. Ripa and P. A. Nilsson, "A Chlamydia trachomatis strain with a 377-bp deletion in the cryptic plasmid causing false-negative nucleic acid amplification tests," Sexually Transmitted Diseases, vol. 34, no. 5, pp. 255-256, 2007.

[99] B. Herrmann, A. Törner, N. Low et al., "Emergence and spread of Chlamydia trachomatis variant, Sweden," Emerging Infectious Diseases, vol. 14, no. 9, pp. 1462-1465, 2008.

[100] B. Herrmann, "A new genetic variant of Chlamydia trachomatis," Sexually Transmitted Infections, vol. 83, no. 4, pp. 253-254, 2007.

[101] J. K. Møller, L. N. Pedersen, and K. Persson, "Comparison of Gen-Probe transcription-mediated amplification, Abbott PCR, and Roche PCR assays for detection of wild-type and mutant plasmid strains of Chlamydia trachomatis in Sweden," Journal of Clinical Microbiology, vol. 46, no. 12, pp. 3892-3895, 2008.

[102] J. K. Møller, L. N. Pedersen, and K. Persson, "Comparison of the Abbott RealTime CT new formulation assay with two other commercial assays for detection of wild-type and new variant strains of Chlamydia trachomatis," Journal of Clinical Microbiology, vol. 48, no. 2, pp. 440-443, 2010.

[103] A. Walsh, F. O. Rourke, B. N. Laoi, and B. Crowley, "Evaluation of the Abbott RealTime CT assay with the BD ProbeTec ET assay for the detection of Chlamydia trachomatis in a clinical microbiology laboratory," Diagnostic Microbiology and Infectious Disease, vol. 64, no. 1, pp. 13-19, 2009.

[104] E. E. Culler, A. M. Caliendo, and F. S. Nolte, "Reproducibility of positive test results in the BDProbeTec ET system for detection of Chlamydia trachomatis and Neisseria gonorrhoeae," Journal of Clinical Microbiology, vol. 41, no. 8, pp. 3911-3914, 2003.

[105] S. Castriciano, K. Luinstra, D. Jang et al., "Accuracy of results obtained by performing a second ligase chain reaction assay and PCR analysis on urine samples with positive or nearcutoff results in the LCx test for Chlamydia trachomatis," Journal of Clinical Microbiology, vol. 40, no. 7, pp. 2632-2634, 2002.

[106] J. Schachter, J. M. Chow, H. Howard, G. Bolan, and J. Moncada, "Detection of Chlamydia trachomatis by nucleic acid amplification testing: our evaluation suggests that CDC-recommended approaches for confirmatory testing are ill-advised," Journal of Clinical Microbiology, vol. 44, no. 7, pp. 2512-2517, 2006.

[107] L. B. Dunham, K. J. Sconce, L. R. Schreck, and D. W. Craft, "Reproducibility of low range positive results for Chlamydia trachomatis and Neisseria gonorrhoeae using GEN-PROBE APTIMA COMBO 2 assay," in Proceedings of the 105th General Meeting of the American Society for Microbiology, Atlanta, Ga, USA, June 2005.

[108] Y. F. Wang, M. Gustafson, T. Triplet, and R. C. Allen, "Evaluation of low positive results from Gen-Probe APTIMA Combo 2 assay for C. trachomatis and N. gonorrhoeae (CT/GC)," in Proceedings of the 105th General Meeting of the American Society for Microbiology, Atlanta, Ga, USA, June 2005.

[109] E. Munson, V. Boyd, J. Czarnecka et al., "Evaluation of GenProbe APTIMA-based Neisseria gonorrhoeae and Chlamydia trachomatis confirmatory testing in a metropolitan setting of high disease prevalence," Journal of Clinical Microbiology, vol. 45, no. 9, pp. 2793-2797, 2007.

[110] J. Schachter, E. W. Hook, D. H. Martin et al., "Confirming positive results of nucleic acid amplification tests (NAATs) for Chlamydia trachomatis: all NAATs are not created equal," Journal of Clinical Microbiology, vol. 43, no. 3, pp. 1372-1373, 2005.

[111] E. M. Peterson, V. Darrow, J. Blanding, S. Aarnaes, and L. M. de la Maza, "Reproducibility problems with the AMPLICOR PCR Chlamydia trachomatis test," Journal of Clinical Microbiology, vol. 35, no. 4, pp. 957-959, 1997.

[112] M. A. Chernesky, D. H. Martin, E. W. Hook et al., "Ability of new APTIMA CT and APTIMA GC assays to detect Chlamydia trachomatis and Neisseria gonorrhoeae in male urine and urethral swabs," Journal of Clinical Microbiology, vol. 43, no. 1, pp. 127-131, 2005.

[113] M. R. Golden, J. P. Hughes, L. E. Cles et al., "Positive predictive value of Gen-Probe APTIMA Combo 2 testing for Neisseria gonorrhoeae in a population of women with low 
prevalence of $N$. gonorrhoeae infection," Clinical Infectious Diseases, vol. 39, no. 9, pp. 1387-1390, 2004.

[114] S. N. Zanto, J. Beebe, E. Hieb, C. Rogers, and Y. Thomas, "Performance of Gen-Probe ASR CT and GC reagents as confirmatory tests for Chlamydia trachomatis and Neisseria gonorrhoeae APTIMA screening-a multistate study," in Proceedings of the 105th General Meeting of the American Society for Microbiology, Atlanta, Ga, USA, June 2005.

[115] C.-Y. Lau and A. K. Qureshi, "Azithromycin versus doxycycline for genital chlamydial infections: a meta-analysis of randomized clinical trials," Sexually Transmitted Diseases, vol. 29, no. 9, pp. 497-502, 2002.

[116] Centers for Disease Control and Prevention, "Sexually transmitted diseases treatment guidelines, 2010," Morbidity and Mortality Weekly Report, vol. 59, no. RR-12, pp. 1-110, 2010.

[117] K. A. Workowski, M. F. Lampe, K. G. Wong, M. B. Watts, and W. E. Stamm, "Long-term eradication of Chlamydia trachomatis genital infection after antimicrobial therapy: evidence against persistent infection," Journal of the American Medical Association, vol. 270, no. 17, pp. 2071-2075, 1993.

[118] C. A. Gaydos, K. A. Crotchfelt, M. R. Howell, S. Kralian, P. Hauptman, and T. C. Quinn, "Molecular amplification assays to detect chlamydial infections in urine specimens from high school female students and to monitor the persistence of chlamydial DNA after therapy," Journal of Infectious Diseases, vol. 177, no. 2, pp. 417-424, 1998.

[119] S. A. Morré, P. T. G. Sillekens, M. V. Jacobs et al., "Monitoring of Chlamydia trachomatis infections after antibiotic treatment using RNA detection by nucleic acid sequence based amplification," Journal of Clinical PathologyMolecular Pathology, vol. 51, no. 3, pp. 149-154, 1998.

[120] A. Bianchi, M. Bogard, G. Cessot, J. M. Bohbot, J. E. Malkin, and J. M. Alonso, "Kinetics of Chlamydia trachomatis clearance in patients with azithromycin, as assessed by first void urine testing by PCR and transcription-mediated amplification," Sexually Transmitted Diseases, vol. 25, no. 7, pp. 366-367, 1998.

[121] J. S. Moran and W. C. Levine, "Drugs of choice for the treatment of uncomplicated gonococcal infections," Clinical Infectious Diseases, vol. 20, supplement 1, pp. S47-S65, 1995.

[122] Centers for Disease Control and Prevention, "Update to CDC's sexually transmitted diseases treatment guidelines, 2006: fluoroquinolones no longer recommended for treatment of gonococcal infections," Morbidity and Mortality Weekly Report, vol. 56, no. 14, pp. 332-336, 2007.

[123] J. W. Tapsall, "What management is there for gonorrhea in the postquinolone era?" Sexually Transmitted Diseases, vol. 33, no. 1, pp. 8-10, 2006.

[124] J. C. Thomas, D. H. Weiner, V. J. Schoenbach, and J. A. Earp, "Frequent re-infection in a community with hyperendemic gonorrhoea and chlamydia: appropriate clinical actions," International Journal of STD and AIDS, vol. 11, no. 7, pp. 461-467, 2000.

[125] J. W. Hanks, C. T. Scott, C. E. Butler, and D. W. Wells, "Evaluation of a DNA probe assay (Gen-Probe PACE 2) as the test of cure for Neisseria gonorrhoeae genital infections," Journal of Pediatrics, vol. 125, no. 1, pp. 161-162, 1994.

[126] L. H. Bachmann, R. A. Desmond, J. Stephens, A. Hughes, and E. W. Hook III, "Duration of persistence of gonococcal DNA detected by ligase chain reaction in men and women following recommended therapy for uncomplicated gonorrhea," Journal of Clinical Microbiology, vol. 40, no. 10, pp. 3596-3601, 2002.
[127] C. Jenny, "Sexually transmitted diseases and child abuse," Pediatric Annals, vol. 21, no. 8, pp. 497-503, 1992.

[128] J. Schachter, M. Grossman, R. L. Sweet, J. Holt, C. Jordan, and E. Bishop, "Prospective study of perinatal transmission of Chlamydia trachomatis," Journal of the American Medical Association, vol. 255, no. 24, pp. 3374-3377, 1986.

[129] T. A. Bell, W. E. Stamm, S. P. Wang, K. K. Holmes, and J. T. Grayston, "Chronic Chlamydia trachomatis infections in infants," Journal of the American Medical Association, vol. 267, no. 3, pp. 400-402, 1992.

[130] R. G. Girardet, S. Lahoti, L. A. Howard et al., "Epidemiology of sexually transmitted infections in suspected child victims of sexual assault,” Pediatrics, vol. 124, no. 1, pp. 79-86, 2009.

[131] P. Kelly and J. Koh, "Sexually transmitted infections in alleged sexual abuse of children and adolescents," Journal of Paediatrics and Child Health, vol. 42, no. 7-8, pp. 434-440, 2006.

[132] P. Kohlberger and D. Bancher-Todesca, "Bacterial colonization in suspected sexually abused ahildren," Journal of Pediatric and Adolescent Gynecology, vol. 20, no. 5, pp. 289-292, 2007.

[133] K. J. Simmons and D. J. Hicks, "Child sexual abuse examination: is there a need for routine screening for $N$. gonorrhoeae and C. trachomatis?" Journal of Pediatric and Adolescent Gynecology, vol. 18, no. 5, pp. 343-345, 2005.

[134] M. R. Hammerschlag, "Use of nucleic acid amplification tests in investigating child sexual abuse," Sexually Transmitted Infections, vol. 77, no. 3, pp. 153-154, 2001.

[135] H. M. Corneli, "Nucleic acid amplification tests (polymerase chain reaction, ligase chain reaction) for the diagnosis of Chlamydia trachomatis and Neisseria gonorrhoeae in pediatric emergency medicine," Pediatric Emergency Care, vol. 21, no. 4, pp. 264-270, 2005.

[136] J. Matthews-Greer, G. Sloop, A. Springer, K. McRae, E. LaHaye, and R. Jamison, "Comparison of detection methods for Chlamydia trachomatis in specimens obtained from pediatric victims of suspected sexual abuse," Pediatric Infectious Disease Journal, vol. 18, no. 2, pp. 165-167, 1999.

[137] R. G. Girardet, N. McClain, S. Lahoti, K. Cheung, B. Hartwell, and M. McNeese, "Comparison of the urinebased ligase chain reaction test to culture for detection of Chlamydia trachomatis and Neisseria gonorrhoeae in pediatric sexual abuse victims," The Pediatric Infectious Disease Journal, vol. 20, no. 2, pp. 144-147, 2001.

[138] N. D. Kellogg, J. Baillargeon, J. L. Lukefahr, K. Lawless, and S. W. Menard, "Comparison of nucleic acid amplification tests and culture techniques in the detection of Neisseria gonorrhoeae and Chlamydia trachomatis in victims of suspected child sexual abuse," Journal of Pediatric and Adolescent Gynecology, vol. 17, no. 5, pp. 331-339, 2004.

[139] C. M. Black, E. M. Driebe, L. A. Howard et al., "Multicenter study of nucleic acid amplification tests for detection of Chlamydia trachomatis and Neisseria gonorrhoeae in children being evaluated for sexual abuse," The Pediatric Infectious Disease Journal, vol. 28, no. 7, pp. 608-613, 2009.

[140] Centers for Disease Control and Prevention, "Sexually transmitted diseases treatment guidelines, 2006," Morbidity and Mortality Weekly Report, vol. 55, no. RR-11, pp. 1-94, 2006.

[141] M. R. Hammerschlag and C. D. Guillén, "Medical and legal implications of testing for sexually transmitted infections in children," Clinical Microbiology Reviews, vol. 23, no. 3, pp. 493-506, 2010. 


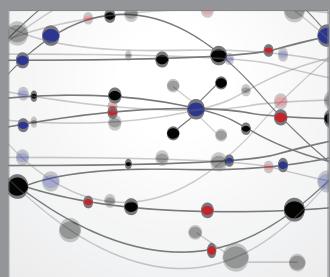

The Scientific World Journal
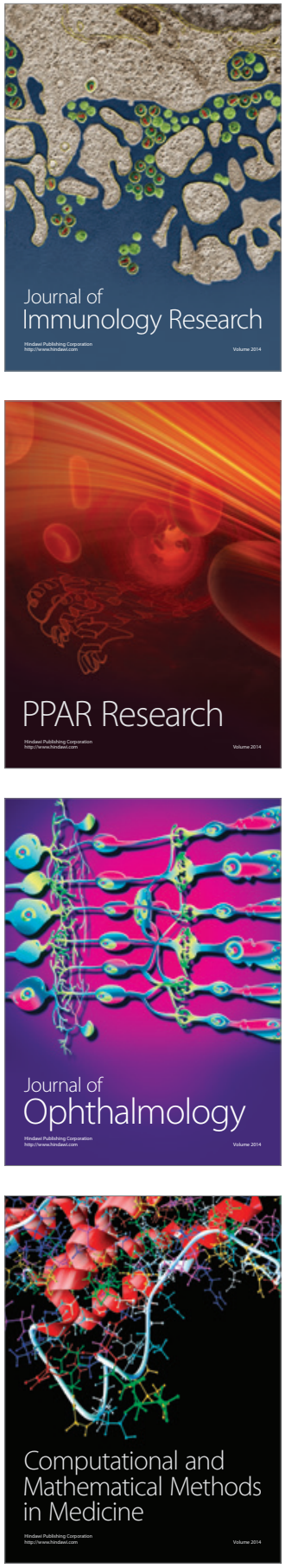

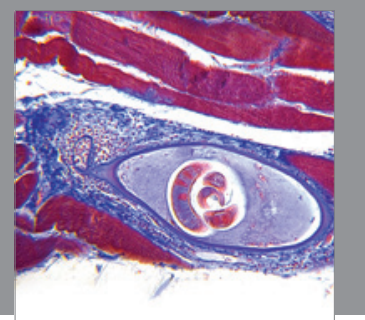

Gastroenterology

Research and Practice
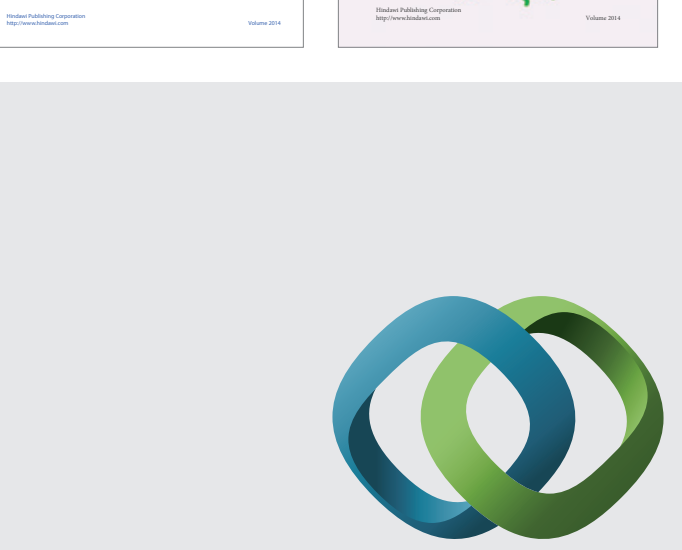

\section{Hindawi}

Submit your manuscripts at

http://www.hindawi.com
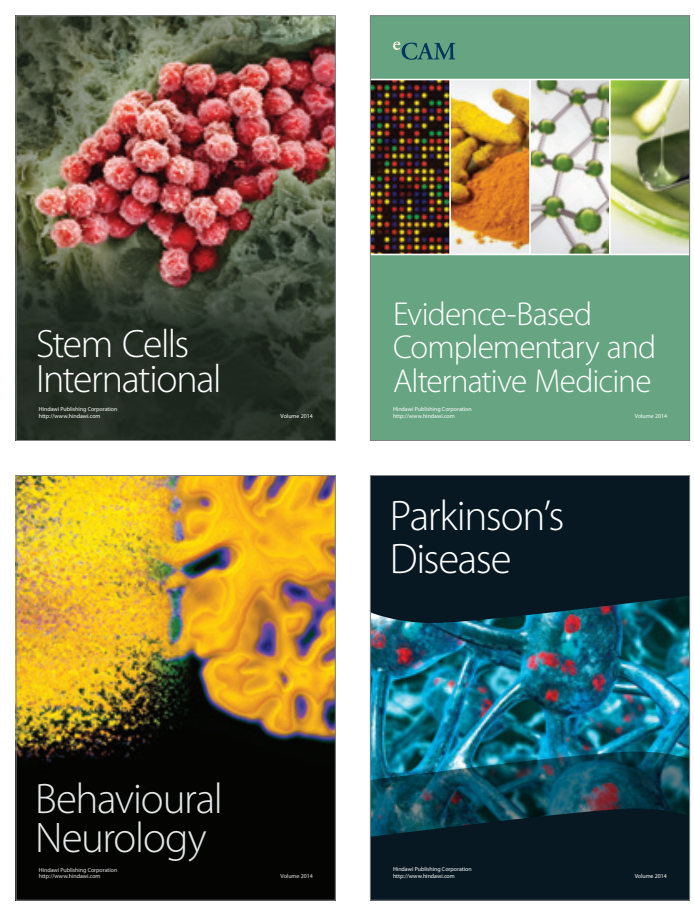

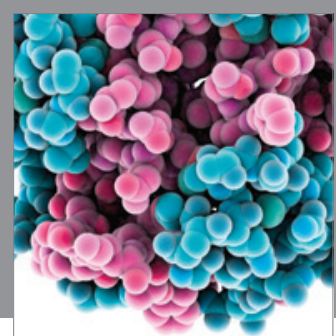

Journal of
Diabetes Research

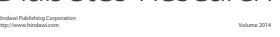

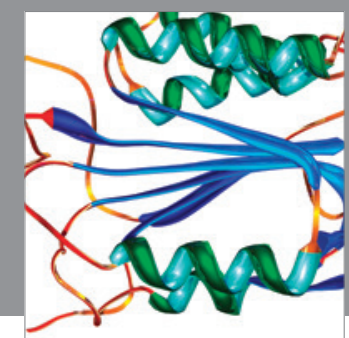

Disease Markers
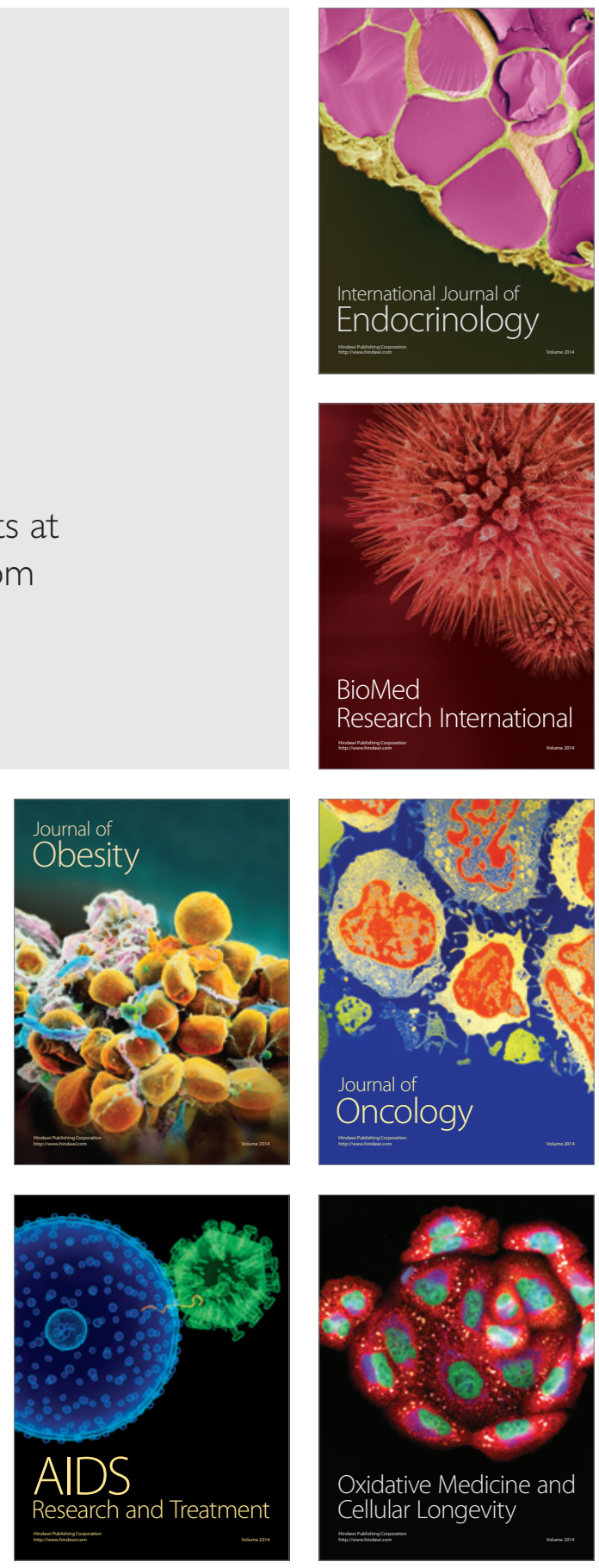